\title{
Surpressions derrière les murs de quai
}

\section{Overpressure behind quay walls}

\author{
par la Professeur Edgar schUL'TZE \\ DIRECTEUR DE L'INSTTUT POUR LXS CONSTRUCTIONS HYDRALLQLES DES VOHES NAYGaBles, \\ LES FONDATIONS ET LA MÉCANIQUE DES SOLS, \\ RATTACHÉ A L'ÉCOLE TECHNIQUE SUPÉRIEURE D'ALX-LA-CHAPELLE
}

\begin{abstract}
Recherche des équations générales régissant les fluctuations de pression dans une nappe derriere un mur de quai, connaissant les fluctuations du niveau libre

- en se limitant à deux dimensions,

- dans le cas simple où les mouvements dans la nappe sont supposés concentrés dans un tube de section constante entourant le mur,

- en supposant que l'écoulement dans la nappe constitue un réseau isotherme.

- dans le cas d'un terrain homogène oll d'un terrain stratifie.

Application ana cas où : le niveant libre est soumis at une marée sinusoüdale; le nivean libre (en rivière) subit une variation simple mais particulierement redontable.

Comparaison des résultats théoriques abec certains résultats de mesures sur des ouvrages réels.

Possibilités et limites de l'étude théorique de ces problemes.
\end{abstract}

\begin{abstract}
Search for general equations governing the pressure fluctuations in a nappe of uater behind a quay wall, the fluctuations of the free surface being known:

- in two dimensions only;

-... in the simple case in which movements in the nappe are hypothetically concentrated in a constant cross-section tube around the wall; - in the supposition that flow in the nappe forms an isothermic flownet;

- in the case of homogeneous or stratified ground.

Application to the cases in which: the free surface is submitted to a sinusoidal tide,

the free surface (of a river) andergoes a simple but particularly feared variation.

Comparison of theoretical results with certain results of measurements made on actual stractures.

Possibilities and limits of the theoretical investigations of these problems.
\end{abstract}

\section{I. - INTRODUCTION}

Avant d'aborder l'étude d'un projet de fondations, il y a lieu d'éclaircir une série de questions qui ont une influence décisive sur les dimensions ultérieures de la section. Parmi ces questions figurent, par exemple, la détermination de la charge admissible sur le sol de fondation et l'importance de la pression hydraulique, ainsi que de la sous-pression. Etant donné que, pour tous les murs qui subissent de telles charges, on doit avoir, avant d'aborder le projel, des indications précises et chiffrées sur la grandeur de ces forces, on pourrait croire qu'on a déjà trouvé, justement du fait de leur fréquence, des réponses précises à ces questions inévitables. Tel n'est malheureusement pas lo eas. Ce sont souvent les problèmes apparemment les plus simples, dont la solution offre le plus de difficulté. Il en est de mème ici. La manière dont on procède encore actuellement pour déterminer la charge admissible sur les fondations et la pression de l'eau, est presque toujours purement empirique. La valeur de l'effort est évaluée plus ou moins grossièrement a l'aide des observations faites et de l'expérience acquise sur les ouvrages voisins. Or, les charges admises dans les calculs d'ouvrage font apparaitre, même dans des cas analogues, une grande diver'sité en ce qui concerne la surpression hydraulique, objet de la présente étude. A quelques exceptions peu nombreuses près, il n'a pas encore été possible, jusqu'ici, 
de fournir à l'Ingénieur projeteur, outre les renseignements sur les conditions locales, un moyen lui permettant de déterminer la valeur de la surpression hydraulique susceptible de se produire derrière un mur de quai. Des incertitudes de deux natures apparaissent dans la détermination des surpressions s'exercant sur les murs de quai adossés au terrain. D'abord on ne comnaît jamais exactement le niveau de la nappe derrière un mur de quai, dont dépend la grandeur de la surpression hydraulique à laquelle il faut ajouter aussi la sous-pression, car, au fond, elle n'est rien d'autre qu'une surpression hydraulique sur des surfaces horizontales au lieu de surfaces verticales. De plus, on trouve encore souvent sur la répartition de la pression hydraulique des opinions qui sont incompatibles avec la mécanique des fluides.

Si l'on cherche, en raison mème de leur fréquence, à rendre solubles ces problèmes si importants, il faut tout d'abord les poser de manière aussi précise que possible, et cela de sorte qu'on puisse avoir recours aux mathématiques. Il s'agit, ici, dans tous les cas, d'applications des lois de l'écoulement en milieu poreux, qui ont déjà été étudiées de manière approfondie dans d'autres domaines, mais qui, jusqu'à présent, n'ont jamais été systématiquement mises à profit dans les problèmes de surpressions.

Ceci est dû, en partie, au fait que la mise en équation de ces problèmes est difficile et implique des simplifications parfois assez importantes. Il faut remplacer les conditions réelles, souvent très compliquées, - ne serait-ce que par suite de la nature stratifiée du terrain, par une représentation simple, dont on ne sait pas toujours a priori si elle suffit entièrement à expliquer les mouvements de la nappe. Mais pour atteindre ce but, on cherchera d'abord à traduire la réalité d'une manière générale par un schéma, que l'on perfectionnera ensuite, s'il le faut. II est un fait que mème une reproduction relativement simple offre l'avantage d'orienter, dans une voie bien définie, les idées souvent très imprécises que l'on se fait des phénomènes hydrauliques en milieu poreux. En outre, on est alors a même d'établir des équations qui, toutefois, ne sont valables, au premier abord, que pour les conditions artificielles correspondant à la représentation choisie. Dans la plupart des cas, il est alors possible de choisir les hypothèses de manière à en déduire des équations avec des solutions de forme maniable. Mais mème une equation différentielle, assez compliquée of difficile à résoudre dans certains cas, peut ètre utile pour l'étude du problème général.

L'intérêt de la solution ne réside pas tellement dans ses indications numériques - après les tâtonnements de la période précédente on ne peut pas s'altendre à trouver d'un seul coup des résultats toụt à fait précis - mais plutôt dans ses indications concernant la nature et linfluence relative des divers facteurs auxquels est lice la surpression. Bien que ces facteurs puissent varier beaucoup d'un cas à un autre, de telles solutions permettent parfois de dégager des lois générales indiquant, sans intervention de calculs, que dans telles ou telles conditions de sol, on peut prévoir telles ou telles variations de la nappe. On restreint ainsi considérablement le domaine des suppositions et on recueille des indications précieuses sur les répercussions de certaines dispositions techniques telles que les drainages. En outre, les renseignements numériques acquis peuvent être utilisés au moins à contrôler l'ordre de grandeur des surpressions; ils peuvent ensuite permettre des comparaisons avec des mesures faites sur l'ouvrage exécuté. Grâce à de telles comparaisons, on peut vérifier la légitimité des hypothèses simplificatrices.

\section{II. - LE NIVEAU DE LA NAPPE DERRIẼRE UN MUR DE QUAI}

\section{a) Première représentation}

Pour calculer la surpression sur un mur de quai, il faut, connaissant les fluctuations du niveau libre, savoir déterminer le niveau de la nappe derrière le mur. Il est surtout intéressant. de savoir préciser les conditions pour lesquelles la dénivellation entre le niveaul libre et la nappe atteint sa valeur maximum : e'est là en effet le cas le plus défavorable du point de vue surpression. Mais avant de considérer ce cas particulier, il y a lieu d'étudier d'une manière générale la variation, dans le temps, de la nappe souterraine en arrière du mur de quai.
Conformément au principe de se faire d'abord, à l'aide d'une représentation aussi simple que possible, une première idée des phénomènes en cause, on remplacera les conditions représentées sur la figure 1 par exemple, derrière un mur de quai sur pieux, par la représentation de la figure 2. Dans le passage de la figure 1 à la figure 2, il importe de garder sans changement les conditions hydrauliques essentielles. La figure 1 est basée sur l'hypothèse que le niveau libre (1) varie suivant une loi,' d'abord arbitraire, en direction de (2) et au-delà. Le niveau de la nappe effectue entre temps un mouvement qui est fonction du précédent et qui est représenté par la dis- 
tance (1) - (3). Dans l'exemple de la figure 1, on a choisi en eflet pour position (1) celle qui correspond justement à la coincidence des deux niveaux. Dès qu'il y a une dénivellation entre les deux niveaux, l'eau de la nappe s'écoule autour du mur suivant une trajectoire représentée schématiquement par (4). La trajectoire commence au plan d'eau instantané de la nappe et aboutit au fond de l'eau libre. Afin de simplifier le calcul, on admet qu'on peut prendre comme plan origine de la trajectoive, invariable durant tout le phénomène, un niveau moyen (5) de la nappe. L'erreur ainsi commise n'est pas trop grande, car la longueur (1) de la trajectoile (4) est, en géne-
1 - Niveau libre initial $=$ niveau initial de la nappe;

2 -..- Niveau libre final;

3 - Niveau de la nappe à la fin de l'abaissement du niveau libre $h$ (instant $\left.t_{1}\right)$;

4 -..- Trajectoire dans la nappe derrière le mur;

5 -.. Nivean moyen de départ des trajectoires;

6-.-Variation du niveau libee en fonction du temps;

7 .... Variation du niveau de la nappe en fonction du temps.
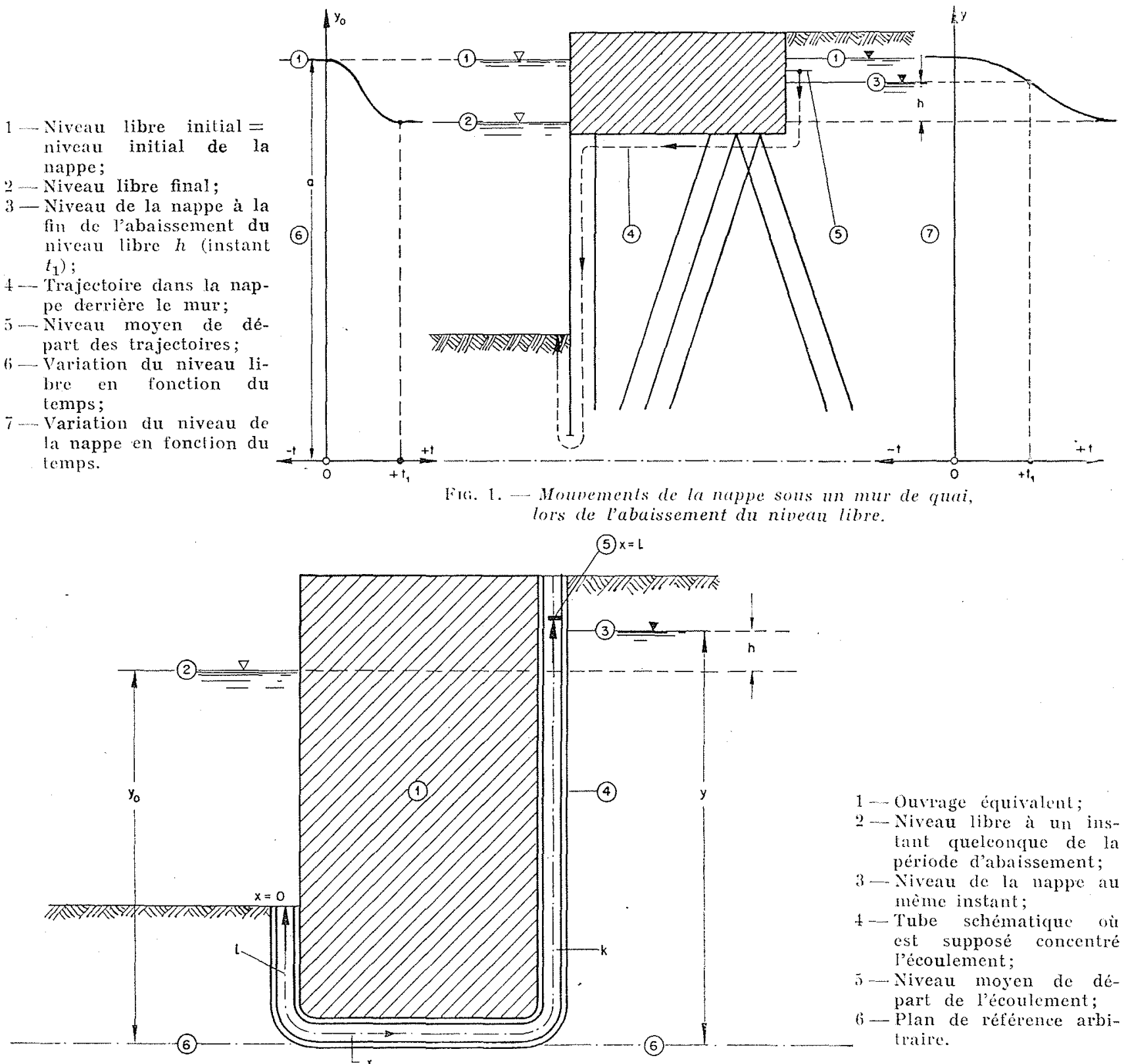

Fla. 2. - Schémalisalion des phénomines

representés sur la fig. 1.

Ial, grande vis-a-bis des fluctuations du nivean de la nappe (entre (1) et (3)) autour du niveau moyen (5). Ce n'est que dans le cas d'une trajectoine $l$ courte, telle qu'elle pourrait s'établir s'il $y$ avait un drainage, que cette erreur devient sensible. 'Toutefois, on n'a pas besoin alors de compter avec une surpression hydraulique, de sorte que tout calcul devient superflu.

La disposition du mur de quá représenté sur la figure 1 a donc été simplifiée sur la figure 2 suivant une première schématisation nécessaire aux considérations ultérieures. Le mur de quai 
est remplacé par un bloc rectangulaire (1) ayant mème périmètre enterré (4) et mème plan origine (5) de la trajectoire $l$. La cote $y_{0}=(2)$ du niveau libre est une donnée, tandis que te niveau de la nappe, à la cote $y=(3)$, et par conséquent la dénivellation $h$ à un moment quelconque, constituent l'inconnue. Les deux cotes $y$ et $y_{0}$ sont rapportées à l'axe de référence horizontal (6), situé arbitrairement. Afin de représenter le phénomène de l'écoulement autour du mur de la manière la plus simple, on considère les variations du niveau à l'intérieur du tuyau que représente la figure 2 et qui est supposé rempli d'un matériau homogène de perméabilité $I$. Ce schéma correspond done au probleme plan qui est d'autant plus près de la réalité que le mur est plus long perpendiculairement à la section.

\section{b) EQuations fondameNTates}

Le problème à résoudre consiste à établir une équation traduisant les fluctuations du niveau $y$ de la nappe dans le tuyau, lorsque les variations du niveau libre $y_{0}$ sont données.

De tels écoulements non permanents en milieu poreux suivent la loi de Darcy qui, sous sa forme la plus générale, -- tout au moins dans la mesure où elle est nécessaire pour les problèmes en question, a pour expression :

$$
J=-\frac{d h}{d x}=\frac{v}{k}+\frac{1}{g}\left(\frac{d v}{d t}+v \frac{d v}{d x}\right)
$$

dans laquelle on désigne par :

$J=$ la perte de charge linéaire dans l'écoulement souterrain.

$h=y-y_{0}=\mathrm{la}$ surpression en $m$ à un instant quelconque $t$.

$y=$ le niveau de la nappe derrière le mur rapporté à un plan de référence horizontal quelconque et exprimé en $\mathrm{m}$.

$v=$ la ritesse d'écoulement de l'eau souterraine en $\mathrm{m} / \mathrm{s}$.

$J_{i}=$ le coefficient de perméabilité du sol en $\mathrm{m} / \mathrm{s}$.

$g=9,81 \mathrm{~m} / \mathrm{s}^{2}$.

$t=10$ temps en s.

$x:=$ la distance en $\mathrm{m}$ mesurée suivant l'axe longitudinal, du tuyau depuis $x=0$ au fond de l'eau libre, jusqu'à $x=l$ au niveau de la nappe (5).

A cette équation de l'écoulement s'ajoute encore l'équation de continuité (fig. 3) :

$$
\frac{d q}{d x}=-\frac{d z}{d t}
$$

dans laquelle :

$z=$ l'axe normal aux $x$ en 11 ;

$q=$ le débit dans le tuyau ou, dans d'autres cas, dans une section arbitrairement choisie de largeur $z_{0}$; ainsi :

$$
v=\frac{d q}{d z}=-\frac{d x}{d t}
$$

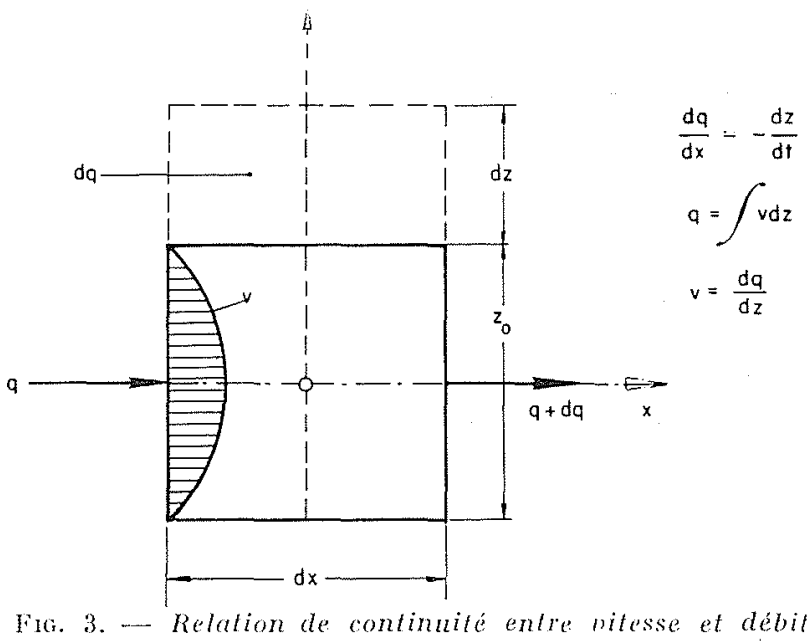

Si la section du tuyau est constante, la vitesse doit être partout la même le long du tuyau. Par conséquent, on a dans l'équation (1), $\frac{d v}{d x}=0 \mathrm{et}$ daus l'équation (2) $\frac{d q}{d x}=0$. De ce fait, et si l'on remplace $d x$ par $l$ et $d h$ simplement par $h$, l'équation (1) se simplifie de la manière suivante :

$$
J=-\frac{h}{l}=\frac{v}{k}+\frac{1}{g} \frac{d v}{d t}
$$

Comme il est toujours possible de s'arranger pour que, dans la dernière branche verticale du tuyau (fig. 2), $x=y$, on peut déduire de l'équation (3a) la relation suivante :

$$
v=-d y / d t
$$

Par conséquent, la vitesse de l'écoulement soulterrain est égale à la vitesse d'abaissement du nivean de la nappe dans le tuyan.

\section{c) EQvation simputiée}

L'équation (4) peut être encore simplifiée, si l’on néglige la variation dáns le temps de la vitesse de l'écoulement souterrain, c'est-à-dire si l'on pose $d v / d t=0$. I'équation sera d'abord résolue dans ce cas. Toutefois, cette variation de la 
vitesse de l'écoulement sera prise en considération ensuite, afin de corriger l'erreur résultant de cette simplification. Une comparaison des résultats montrera alors que la simplification de l'équation (4) se justifie.

On obtient donc avec cette hypothèse, pour l'écoulement souterrain, l'équation connue de DARCY :

$$
v=k . \mathrm{J}
$$

La perte de charge linéaire peut être exprimée par l'équation :

$$
\mathbf{J}=h / l=\left(y-y_{0}\right) / l
$$

En raison de la fréquence du facteur $k / l$, on pose pour simplifier :

$$
k / l=\alpha
$$

En introduisant les expressions indiquées, 5, 7 et (8 a) dans l'équation (6), on obtient l'équation différentielle pour l'écoulement de l'eau souterraine dans le tuyau (4) de la figure 2.

$$
d y / d t+x y=\alpha y_{0}
$$

\section{d) Solution numérique}

Avant de chercher la solution précise de cette équation, on étudiera d'abord sa forme et les conclusions qui en découlent. Il est évident qu'on ne peut trouver une solution précise que si les fluctuations dans le temps du niveau libre, donc la fonction $y_{0}$ de la figure 1 , sont données par une courbe susceptible d'une interprétation mathématique. Mais dans la plupart des cas, ces fluctuations sont irrégulières. Alors le meilleur. moyen pour intégrer l'équation (9) est de le faire graphiquement, par la méthode des différences finies. Pour cela on pose :

$$
\begin{aligned}
\Delta y & =y_{2}-y_{1} \\
\Delta t & =t_{2}-t_{1} \\
y & =\left(y_{1}+y_{2}\right) / 2
\end{aligned}
$$

On désigne ici (fig. 4) par :

$y_{1}$ : le niveau de la nappe derrière le mur $\mathrm{c}$ un instant quelconque $t_{1}$.

$y_{2}$ : le niveau de la nappe derrière le mur à un instant ultérieur $t_{2}$.

$y$ : la moyenne arithmétique de $y_{1}$ et $y_{2}$.

Si l'on introduit ces valeurs dans l'équation différentielle 9, on obtient l'expression suivante de $y_{2}$ :

$$
y_{2}=\frac{y_{1}(1-\mathrm{A})+2 \mathrm{~A} y_{\mathrm{om}}}{(1+\mathrm{A})}
$$

dans laquelle (fig. 4) :

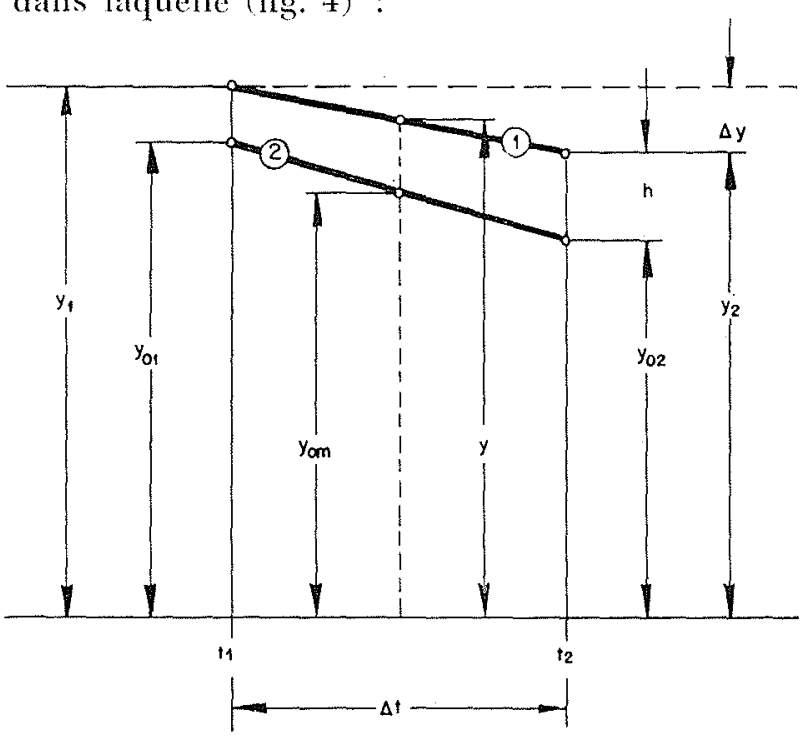

Fra. 4. - Calcul du nivean de la nappe par la methode des differences finies.

1 - Variation cherchée du niveau de la nappe; 2-Variation donnée du niveau libre.

$\mathrm{A}=\alpha \Delta t / 2$

$y_{o m}=\left(y_{o 1}+y_{o 2}\right) / 2$;

$y_{n 1}=$ le niveau libre devant le mur à l'instant $t_{1}$;

$y_{02}=$ le niveau libre à l'instant $t_{2}$.

Si l'on connaìt $y_{1}$, l'équation (11) permet de calculer la cote $y_{2}$ de la nappe. Afin de pouvoir appliquer l'équation, il faut donc connaître, à un instant donné quelconque, la cote du niveau libre et celle de la nappe. C'est d'abord le cas lorsque le niveau libre est resté invariable depuis assez longtemps. Alors, pour les sols dans lesquels les fluctuations de la nappe dépendent, en général, de celles du niveau libre, on peut admettre que le niveau de la nappe se trouve à la mème cote. Ainsi, dans la figure 5 , on a admis qu'au départ (1) la cote $y$ de la nappe et la cote $y_{0} \mathrm{du}$ niveau libre coincidaient. On a alors $y_{1}=y_{01}$. Comme le niveau libre $y_{o m}$ correspond à la valeur moyenne de $y_{0}$ dans l'intervalle de temps considéré, toutes les grandeur's du membre de droite de l'équation (11) sont connues et, par conséquent, on peut calculer $y_{2}$. Dès que cela est fait, le procédé peut être répété pour l'intervalle de temps suivant : le $y_{2}$ calculé devient $y_{1}$ et l'on en déduit un nouveau $y_{2}$, et ainsi de suite.

Ainsi, pour chaque point d'une courbe quel- 
conque mais connue, donnant les variations $d u$ niveau libre, on peut calculer le point correspondant de la courbe, inconnue, des variations du niveau de la nappe (fig. 5). Plus les intervalles de temps $\Delta t$ sont choisis petits, plus la solution est précise.

Un examen de la figure 5 montre qu'au cours du temps, le nivenu libre et celui de la nappe coïncident à plusieurs reprises, mais à des cotes différentes. Par conséquent, on ne peut pas dire, en général, que pour un niveau libre déterminé, par exemple, le niveau moyen, le niveau de la nappe est à la même cote; mais la cote de coïncidence, exclusivement lice à la variation dans le temps du niveau libre, est imprévisible el tout à fait fortuite. Par conséquent, les surpressions

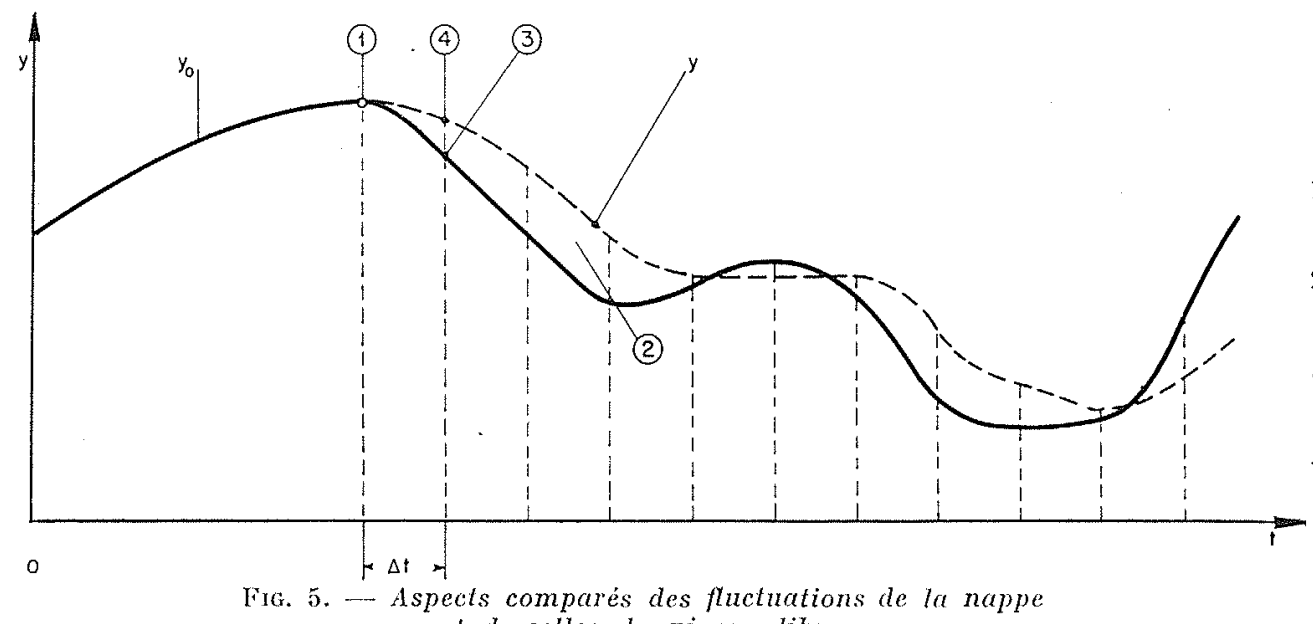

1 - Instant où le niveau libre et celui de la nappe coincident;

2 - Surpression $=($ niveau $y$ de la nappe) - (niveau libre $\left.y_{0}\right)$;

3 - Point donné sur la courbe de variation du niveau libre;

4 -Point calculé sur la courbe de variation du niveau de la nappe. it de celles du niveau libre.

qui agissent sur l'ouvrage dépendent également des fluctuations de niveau libre. Si, par exemple, on avait choisi sur la figure 5 , comme point de départ de calcul, un autre point de coïncidence (1), le niveau $y$ de la nappe d'après l'équation (11) et, par suite, la grandeur de la surpression auraient varié de manière tout à fait différente. On peut dire seulement que, quelque soil la cote de coïncidence de départ (1), les fluctuations de la nappe doivent être telles que son niveau moyen corresponde au niveau moyen libre. Mais cela aussi n'est exact qu'en l'absence de toute alimentation ou de tout écoulement en provenance ou en direction de la zone d'influence de la rivière. En effet, dans de telles circonstances, le mur de quai s'oppose à l'écoulement horizontal et il s'établit une dénivellation entre les niveaux moyens de part et d'autre de l'ouvrage.

\section{e) INTÉGRATION DE L'ÉQUATION GẺNÉraLE}

La solution de l'équation (9) est (1) :

$$
y=e^{-\alpha t}\left(\mathrm{C}+\alpha \int y_{0} e^{\alpha t} d t\right)
$$

La transformation ultérieure de cette équation dépend du choix de la fonction $y_{o}$. Comme celleci se présente différemment suivant qu'il s'agit d'un ouvrage à la mer (marée) ou d'un ouvrage intérieur, nous examinerons plus loin diverses solutions particulières.

Si maintenant l'on considère l'équation complète (4) et si l'on tient compte de (5) et de (6), on aboutit à l'équation différentielle suivante :

$$
\frac{k}{g} \frac{d^{2} y}{d t^{2}}+\frac{d y}{d t}+\frac{k}{l} y=\frac{k}{l} y_{o}
$$

Selon une simplification analogue à celle de l'équation $(8 \alpha)$, on peut encore poser :

$$
k / g=\beta
$$

La solution (2) de l'équation (13) dépend de la valeur du déterminant :

$$
\mathrm{D}=1-4 * \beta=1-4 k^{2} / g l .
$$

Etant donné qu'on a toujours $k$ inférieur à $1 \mathrm{~m} / \mathrm{s}, g=9,81 \mathrm{~m} / \mathrm{s}^{2}$ et $l$ supérieur à $1 \mathrm{~m}$, le déterminant $D$ est positif. On est donc dans le domaine apériodique et la solution est la suivante :

$$
\begin{aligned}
& y=\mathrm{C}_{1} e^{\chi_{1} t}+\mathrm{C}_{2} e^{\varkappa_{2} t}+\frac{\alpha}{\sqrt{1-4 \alpha \beta}} \\
& {\left[e^{\chi_{1} t} \int y_{0} e^{-\varkappa_{1} t} d t-e^{\chi_{2} t} \int y_{0} e^{-x_{2} t} d t\right]}
\end{aligned}
$$

(1) HuTtre, I, $25^{\circ}$ édition, p. 85 ou Schrficher, Taschenbuch für Baningenieure, Berlin, 1943, p. 35.
(2) ScHuerchen. - Taschenbuch fïr Baningenieure, Berlin, 1943, p. 34. 
Cette solution comporte :

- deux termes exponentiels exprimant les oscillations propres de la nappe et diminuant avec le temps;

- un terme représentant les oscillations forcées de la nappe.

Ici aussi la solution complète de l'équation n'est possible que si $y_{0}$ est une fonction bicn déterminée, comme cela a lieu dans les cas particuliers envisagés plus loin.

\section{f) Cas des terrains strattifiés}

Les équations (12) et (15) ont été établies en supposant le tuyau de la figure 2 rempli sur toute sa longueur par un même matériau. Dans le cas où le matériau comporte deux couches distinctes de perméabilité différente, le coefficient $\alpha$ devient :

$$
\alpha=\frac{1}{l_{1} / k_{1}+l_{2} / k_{2}}=\frac{1}{1 / \alpha_{1}+1 / \alpha_{2}}
$$

ou, d'une manière générale, pour $i$ couches de sol différentes :

$$
\alpha=1 / \Sigma\left(1 / \alpha_{i}\right)
$$

\section{g) Représentation améliorée}

Le schéma, considéré jusqu'à présent d'un tuyau de section constante, ne reproduit les conditions réelles que d'une manière insuffisante, car l'écoulement dans la nappe ressemble plutôt au tracé des lignes de courant de la figure 6 . Si l'on trace le réseau isotherme correspondant, le débit d'écoulement entre deux lignes de courant voisines (6) est une constante $q$. Si l'on admet que le tuyau employé jusqu'ici représente la première ligne de courant immédiatement derrière l'ouvrage, un coup d'œil sur la figure 6 montre qu'il ne peut pas être question d'un tuyau à section constante. On peut se demander si la représentation par un tuyau adoptée jusqu'ici ne pourrait pas être améliorée en utilisant l'image bien meilleure d'un réseau isotherme, bien que ceci ne constitue encore, par rapport à la réalité, qu'une approximation. Le maniement des réseaux isothermes appelle certaines considérations développées ci-après.

\section{h) Coefficient de forme}

Dans un écoulement symétrique en milieu poreux, par exemple autour d'un rideau de palplanches (fig. 7), de part et d'autre duquel le terrain est submergé (3)), les vitesses, au débouché dans le bassin aval, varient suivant une expression de la forme :

$$
v=\operatorname{lin} f(z)
$$

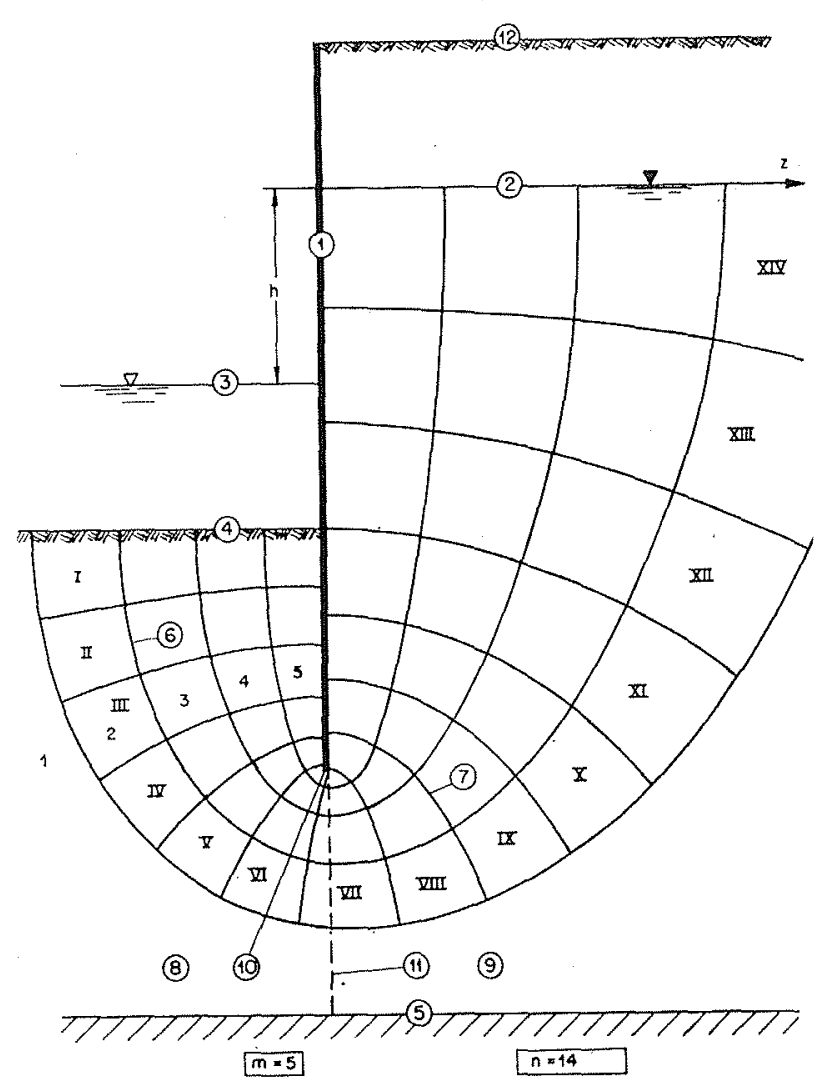

Fia. 6. - Réseau non symétrique atl-dessous d'un rideau de palplanches.

1 - Rideau de palplanches;

2 - Niveau de la nappe;

3 - Niveau libre;

4 - Fond du bassin d'eau libre;

5 - Limite supérieure de la couche relativement imperméable (argile);

6 - Lignes de courant;

7 -Equipotentielles;

8 - Partie gauche du réseau;

9-Partic droite du réseau;

10 - Bord inférieur du rideau de paiplanches;

11 - Ligne de démarcation entre les deux parties (8) et (9) du résean.

On peut rapprocher cette équation de l'équation $(6 a)$ que l'on peut d'ailleurs écrire :

$$
v=k h 1 / l
$$

L'expression (16 $a$ ) a la même forme que celle que l'on tire des équations 6-8 pour un tuyau.

(3) Hofpmann (R.). - Grundwasserströmung unter Wehren, Die Wasserwirtschaft, 27 (1934), $\mathrm{n}^{\circ} 18 / 19$ et 20/21, pp. 174 et 198, fig. 5. 
La fonction $f(z)$ est comnue dans le cas d'un réseau symétrique.

De plus, pour le débit tolal qui passe au-dessous d'un tel rideau de palplanches, on peut icrire :

$$
q=k h \mathrm{~F}
$$

F définissant le coefficient de forme du réseau isotherme considéré.

Les équations (16) et (17) sont reliées entre elles par la relation suivante tirée de l'équation (3a) :

$$
q=\int_{0}^{\infty} v d z
$$

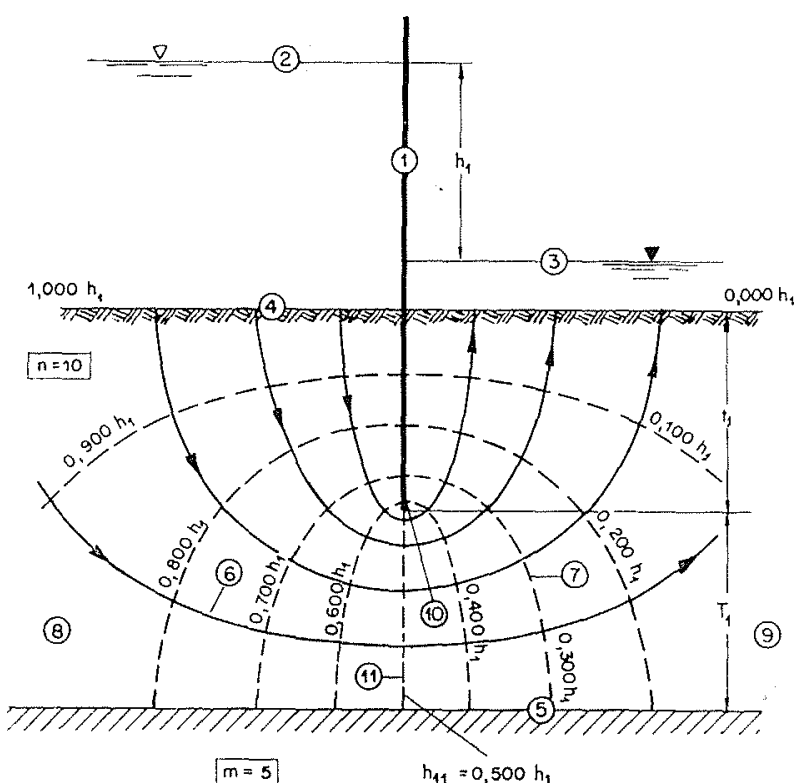

Fia. 7. - Réseau symétrique au-dessous d'un ridean de palplanches.

1 - Rideau de palplanches;

2 - Niveau libre;

3 - Niveau de la nappe (au-dessus du fond du bassin d'eau libre);

4 - Fond du bassin d'ea libre:

5 - Limite supérieure de la couche relativement imperméable (argile)

6-Ligne de courant;

7 - Equipotentielle;

8 - Partic gauche du réseau;

9)- Partic droite du réseau;

10 - Bord inférieur du rideau de palplanches;

11 - Ligne de démarcation entre les deux parties (8) el (9) du résean.

$h_{1}=$ surpression tolate;

$h_{11}=0,5 h_{1}=$ perte de charge dans la zone (8);

$h_{12}=h_{1}-h_{11}=0,5 h_{1}=$ perte de charge dans la zone. (9);

$t_{1}=$ fiche du rideau de planche;

$\mathrm{T}_{1}=$ hautem de la section de passage sous le rideau de palplanches;

$q=k h_{1} F_{1}=2 k h_{11} F_{1}=2 k h_{12} F_{1}=$ débit de tuite sous le rideau de palplanches;

$F_{1}=m / n=1 / 2 \sqrt[2]{T_{1} / t_{1}}=$ coefficient de forme du réseau; $m=$ nombre de tubes de courant du réseau;

$n=$ nombre d'intervalles d'équipotentielles de l'ensemble du réseau.
Le coefficient de forme est donné par l'expression :

$$
\mathbf{F}=m / n
$$

où :

$m$ est le nombre de tubes de courant du réseau isotherme (par exemple 5, sur la figure 7).

$n$ est le nombre d'intervalles d'équipotentielles du réseau (par exemple 10, sur la figure 7 ).

Le coefficient de forme $m / n$ est constant pour toute section de l'ouvrage et pour tout niveau de la nappe souterraine et est une particularité du réseau isotherme considéré. Il dépend done de la forme géométrique du réseau; autrement dit, il constitue une façon simple de caractériser un tel réseau. Si, conformément à la figure 7 , on considère seulement une moitié d'un réseau symétrique (à droite ou à gauche du rideau de palplanches), $m$ reste invariable, mais $n$ se trouve aussi réduit de moitié. Chacun des deux réseaux partiels a donc pour coefficient de forme :

$$
\mathrm{F}_{1}=\mathrm{F}_{2}=2 \mathrm{~F}
$$

Par conséquent, un réseau symétrique de coefficient $\mathrm{F}$ comporte deux demi-réseaux de coéfficient 2 F. En ce qui concerne la perte de charge, et par raison de symétrie, chacun des deux demiréseaux correspond à une hauteur $h_{1}=h_{2}=h / 2$, soit la moitie de la perte de charge du réseau complet. Quant au débit $\left(q_{1}=h_{1} \mathrm{~F}_{1} ; q_{2}=h_{2,} \mathrm{~F}_{2}\right)$ il est évidemment le même, qu'il s'agisse des deux demi-réseaux ou du réseau complet $(q=h \mathrm{~F})$.

\section{i) RÉseaUx yon symétrioues}

Dans l'étude des surpressions, il ne s'agit pas de réseaux symétriques, mais bien de réseaux non symétriques, comme c'est le cas pour le rideau de palplanches de la figure 6. Etant donné qu'il est extrêmement laborieux de tracer chaque fois le réseau particulier correspondant au eas envisagé, il est commode de se reporter (d'après DAchler (t) aux réseaux symétriques et d'en modifier les résultats en conséquence. Comme l'indique Kastrer (..), dans un réseau non symétrique on peut admettre que chacun des deux demi-réseaux distincts dont il est constitué, coüncide approximativement avec une moitié du réseau symétrique correspondant. On peut donc.

(1) Dachuer. - Grunduasserströmung, Vienne, 1936, pD, $55-81$

(5) Kastwer. - Lber die Stundsicherheit bon Spundmänden in strömenden Grundwasset, Bantechnik, 21 (1943), p. 66 . 
Lracer le réseau de la figure 6 en raccordant, dans laxe du rideau de palplanches, deux demi-réseaux dont chacun est une moitié d'un réseau symétrique du type de la figure 7 et dont seul varie le coefficient de forme $\mathrm{F}$.

Si l'on remplace le réseau symétrique de la figure 7 qui correspond à une fiche $t_{1}$ du rideau de palplanches et à une surpression $h_{1}$ par le réseau non symétrique de la figure 6 caracterisé

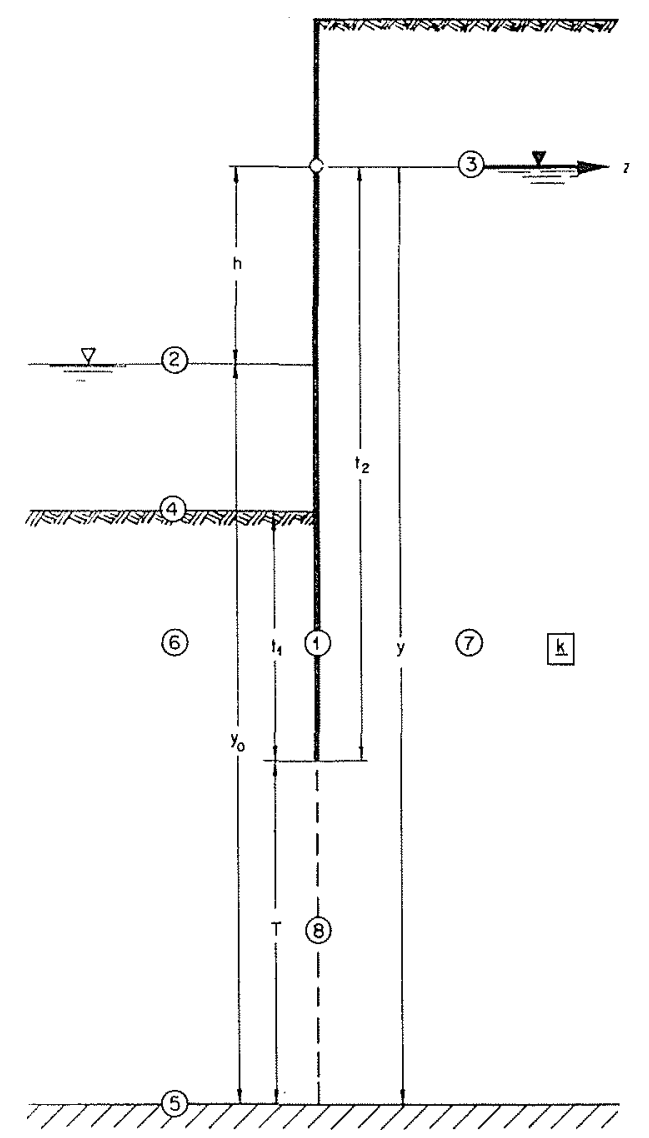

Fic. 8. .-. Gondilions aur limites dans an résean non-symetrique sous an ridean de palplanches.

1 -..-Rideau de palplanches;

2 -Niveau libre;

3 - Niveau de la nappe;

t-Fond du bassin d'eau libre:

5 - Limite supérieure de la couche redativement imperméable (argite);

6-- Partie gatuche du résean;

7 -. Partic droite du réseatu;

8 - Ligne de démarcation entre les deux zoncs correspondant a la perte de charge $h_{11}$;

$\mathrm{T}=$ hauteur de la section de passage sous le rideau de palplanches;

$h=$ surpression;

$k=$ perméabilité du lerrain:

$l_{1}=$ fiche du rideau de palplanches:

$t_{2}=$ hateur de la nappe au-dessus du bord inférieur du rideau des palplanches;

$y=$ hatuteur de la nappe atu-dessus du plan de réference:

$y_{0}=$ cote du nireau hbre an-dessus du plan de reférence, par $t_{1}$ et $t_{2}$ (ig. 8), on peut décomposer la surpression globale $h$ de la manière suivante :

$$
h=h_{1}+h_{2 .} ; h_{1} \text { correspondant à } t_{1} \text { et } h_{2: \text { à }} t_{2 .} \text {. }
$$

Comme le débit reste le mème tout le long du réseau, on peut écrire, conformément aux équations (16) et (17), pour l'écoulement non symétrique :

$$
q=k h \mathrm{~F}=h h_{1} \mathrm{~F}_{1}=k \cdot h_{2} \mathrm{~F}_{2}
$$

formule où :

$F$ est le coefficient de forme de l'ensemble du réseau,

$\mathrm{F}_{1}$ est le coefficient de forme du premier réseau partiel, c'est-à-dire le coefficient de forme du demi-réseau situé à gauche du réseau de palplanches et déterminé en supposant que le demi-réseau de droite en est le symétrique (fig. 11).

$F_{2}$ est le coefficient de forme du deuxième réseau partiel, c'est-à-dire le coefficient de forme du demi-réseau situé à droite du rideau de palplanches et déterminé en supposant que le demi-réseau de gauche en est le symétrique (fig. 12),

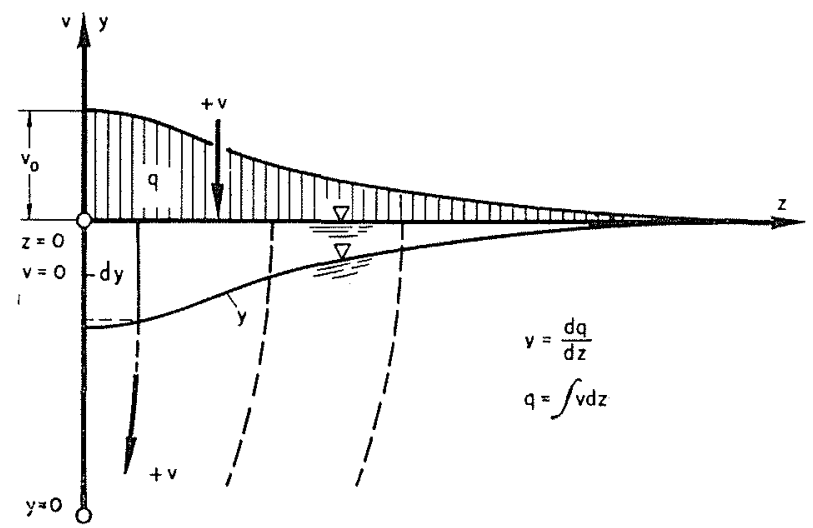

Fiti. 9.-... Profil de la nappe.

$q=$ dobit total au-dessous de l'ouvrage;

b = vitesse d'écoulement à la surface de la nappe;

$y=$ niveau de la nappe;

$z=$ distances comptess nomatement au quai.

$h_{1}$ est la perte de charge du premier réseau partiel, c'est-à-dire la perte de charge correspondant à la région à gauche de l'axe du rideau de palplanches,

h., est la perte de charge du deuxième réseau partiel, c'est-àdire la perte de charge correspondant à la région à droite de l’axe du rideau de palplanches. 
Si $p$ réseaux partiels constituent le réseau d'ensemble, on a, d'une manière générale :

$$
q=k h \mathrm{~F}=k h_{i} \mathrm{~F}_{i} \quad i=1,2,3, \ldots p
$$

On a donc :

$$
h_{i} / h=\mathrm{F} / \mathrm{F}_{i}
$$

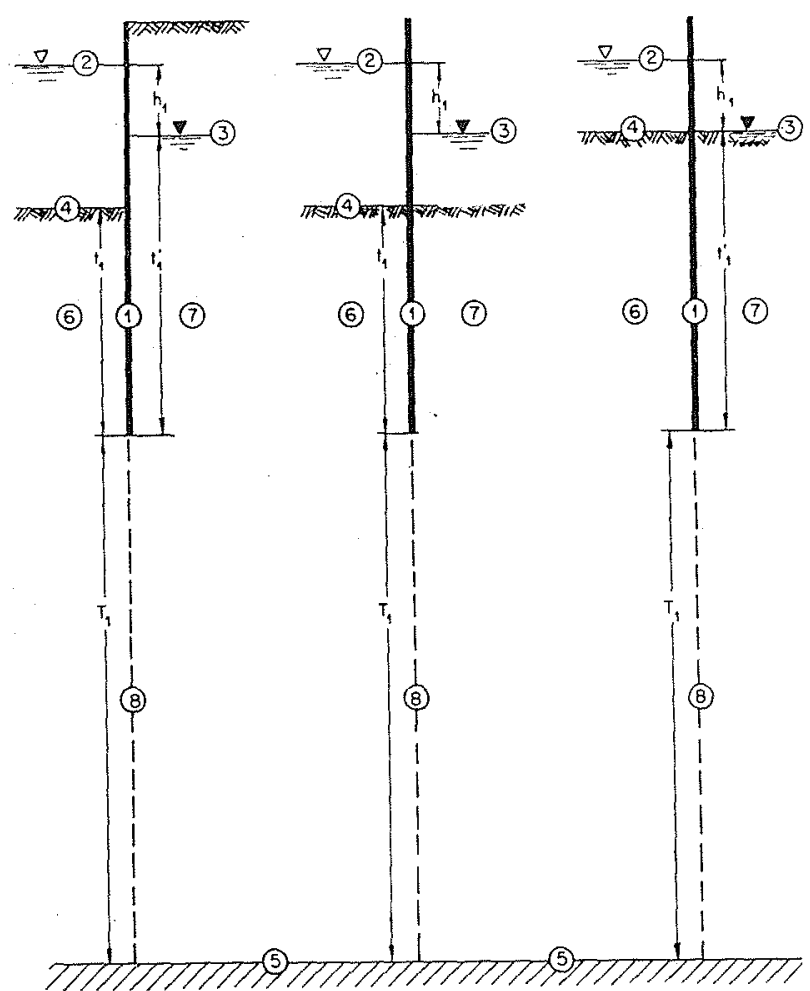

FIG. 10, 11, 12. - Conditions aux limites

pour différentes dispositions d'un réseau non symétrique sous un rideau de palplanches.

1 - Rideau de palplanches;

2 - Niveau libre;

3 - Niveau de la nappe;

4 - Fond du bassin d'eau libre;

5 - Limite supérieure de la couche relativement imperméable (argile);

(i-- Partie gauche du réseau;

7 - Partie droite du réseau;

8-- Ligne de démarcation entre les deux zones correspondant a la perte de charge $h_{11}$;

$h_{1}=$ surpression totale;

$t_{1}=$ fiche du rideau de palplanches;

$\mathrm{T}_{1}=$ hauteur de la section de passage sous le rideau de palplanches;

$t^{\prime}{ }_{1}=$ hauteur de la nappe au-dessus du bord inférieur du rideau de palplanches.

En outre, puisque $m$ est constant, on a :

$$
\mathrm{F}=m / \Sigma_{p} n_{i}=1 / \Sigma_{p} \frac{n_{i}}{m}=1 / \Sigma_{p}\left(1 / \mathrm{F}_{i}\right)
$$

Conformément à l'équation (16 $a)$, on a :

$$
v=2 k h_{p} f(z)
$$

car $h_{p}$ ne s'entend maintenant que pour la moitié du réseau symétrique et non, comme $h$, pour l'ensemble. Par conséquent, il faut poser :

$$
h=2 h_{p}
$$

En introduisant l'équation (20), on obtient :

$$
v=2 k h \frac{\mathrm{F} f(z)}{\mathrm{F}_{p}}
$$

où :

$h$ est la perte de charge totale, c'est-à-dire la surpression,

$h_{p}$ est la perte de charge correspondant au dernier réseau partiel délimité par le niveau de la nappe,

$v$ est la vitesse de sortie de l'eau souterraine à la distance $z$ de l'ouvrage, c'est-à-dire la vitesse de montée ou de descente du niveau de la nappe,

$f(z)$ correspond à la répartition des vitesses le long de $z$ et dépend de la forme du réseau,

$F$ est le coefficient de forme de l'ensemble du réseau

$\mathrm{F}_{p}$ est le coefficient de forme du dernier réseau partiel délimité par le niveau de la nappe.

\section{k) LA LONGUEUR Fictrve}

En égalant les équations $(6 b)$ et $(16 c)$ on trouve la valeur de la longueur fictive à adopter pour le tuyau schématisant le réseau :

$$
l=\frac{\mathrm{F}_{n}}{2 \mathrm{~F} f(z)}=\frac{\mathrm{F}_{p} \Sigma_{y}\left(1 / \mathrm{F}_{i}\right)}{2 f(z)}
$$

On aboutit à la conclusion aue, dans le passage au réseau isotherme, senle la lonquenr l. ef par suite la constante a varient. Par conséanent, les solutions 12 et 15 des éanations différentielles (4) et (9) demenvent valables. Il faut seulement introduire une autre valeur pour $\alpha$. Le fait do considérer $F, F_{n}$ et $f(z)$ conme des constantes s'appuie sur la même hypothèse que celle aui a été adoptée dans l'établissement des équations concernant le tuyau, à savoir fue les variations du plan d'eau derrière le mur sont faibles vis-àvis de la longueur du traiet $l$ (voir p. 811). Fn fait, ces facteurs dépendent de $\underline{m}$ : mais à la puissance $1 / 3$ seulement pour $F_{n}$ et $F$, comme on le montrera plus loin à propós du rideau de palplanches. Ce résultat se retrouve sur d'autres ouvrages. Par conséquent. c'est une approximation valable que de considérer les coefficients de forme comme des constantes dans le temps, lors- 
que les variations du niveau de la nappe ne sont pas trop grandes. Il en est de même pour la fonction $f(z)$. De plus, il faut tenir compte de ce que, pendant l'écoulement, la surface de l'eau n'est plus, comme au départ, horizontale mais s'abaisse vers le mur suivant la courbe $y$ (fig. 9). Tant que la ligne d'abaissement n'a pas une courbure trop grande, on peut l'assimiler, comme on l'a fait jusqu'à présent, par approximation, à une droite, de sorte que la fonction $f(z)$ pent être considérée, de ce point de vue également, comme une constante.

\section{l) Application a un ridenu de patplanches}

Les fonctions $F$ et $F_{i}$, de même que $f(z)$ dépendent de la forme de l'ouvrage en cause. Dans le cas simple d'un rideau de palplanches vertical, Dacmuen (6) propose une formule approchée due à Terzagh donnant le coefficient de forme du réseau symétrique (fig. 7 ); lorscu'on a $t<\mathrm{T}$ :

$$
\mathrm{F}=\frac{1}{2} \sqrt[3]{\mathrm{T} / t}
$$

où :

$t$ - est la fiche du rideau de palplanches (désignée par $t_{1}$ sur la figure 7 ),

T est la hauteur de l'extrćmité inférieure du rideau de palplanches au-dessus de la limite supérieure de la couche imperméable (désignée par ' $\mathrm{T}_{1}$ sur la figure 7 ).

On peut en tirer, d'après l'équation (21), le coefficient de forme pour un réseau non symétrique (fig. 10). On a pour le réseau divisé en deux, conformément à la remarque de la page 17 : $\mathrm{F}_{1}=\sqrt[3]{\mathrm{T} / t_{1}}$ (fig. 11 avec $\mathrm{T}_{1}$ an lien de $\left.\mathrm{T}\right) .(24 a)$ $\mathrm{F}_{2}=\sqrt[3]{\mathrm{T} / t_{2}}\left(\right.$ fig. 12 avec $\mathrm{T}_{1}$ an lieu de $\mathrm{T}$ et $t_{1}$ au lieu de $t_{2}$ ).

Tandis que, dans le cas d'un réseau symétrique, on a le même coefficient de forme et les mêmes pertes de charge partielles de part et d'autre du rideau de palplanches, il n'en est pas de même pour un écoulement non symétrique.

Dans l'écoulement au-dessous d'un rideau de palplanches, les coefficients de forme dépendent des longueurs $\mathbf{T}, t_{1}$ et $t_{2}$ correspondant à $\mathrm{T}_{1}$, $t_{1}$ et $t_{1}^{\prime}$ sur la figure 10 .

En introduisant les équations $(24 a)$ et $(24 b)$ dans l'équation (21); on obtient le coefficient de forme de l'ensemble du réseau non symétrique.

$$
\mathrm{F}=\frac{1}{\sqrt[3]{t_{1} / \mathrm{T}}+\sqrt[3]{t_{2} / \mathrm{T}}}=\frac{\sqrt[3]{\mathrm{T}}}{\sqrt[3]{t_{1}}+\sqrt[3]{t_{2}}}
$$

(6) Dachuer. - Grundwasserströmung, Vienne, 1936, p. 68 , èq. 48. $l_{y}$, qui permel de calculer $\mathrm{F}_{2}$ (ćq. $24 b$ ), puis $i(z)$, correspond au niveau moyen, escompté, de la nappe durant le mouvement considéré (voir fig. $1(5)$ et $2(5))$. La fonction $f(z)$ a été donnée (le manière approchée par Dachler (6) et de manière précise par HorrmanN (i). Il n'est pas utile d'en donner ici l'expression complète, car elle ne sert que pour le calcul de la ligne d'abaissement de la nappe derrière le mur de quai, et non pour celui de la surpression qui nous intéresse et qui ne fait intervenir que sa valeur pour $z=0$. En introduisant cette valeur, on tire de l'équation de Dichlen l'expression suivante :

$$
f(z=0)=\mathrm{F}_{v}=\frac{1}{4\left(\mathrm{~T}+t_{2}\right) \sin \frac{\pi t}{4\left(\mathrm{~T}+t_{2}\right)}}
$$

La vitesse $v$ immédiatement derrière le rideau de palplanches ne dépend donc d'après cela que les valeurs $T$ et $t_{2}$. Elle peut ètre donnée une fois pour toutes par un graphique (fig. 13). On a, d'après Dachler :

$\begin{array}{cc}t /(\mathrm{T}+t) & (\mathrm{T}+t) \mathrm{F}_{v} \\ 0,1 & 3,200 \\ 0,2 & 1,600 \\ 0,3 & 1,075 \\ 0,4 & 0,810 \\ 0,5 & 0,640 \\ 0,6 & 0,488 \\ 0,7 & 0,394 \\ 0,8 & 0,316 \\ 0,9 & 0,243\end{array}$

On peut aussi calculer la vitesse $p$ directement d'après les équations $(16 c)$ et $(26)$ :

$$
v=2 h i h \mathrm{~F} \mathrm{~F}_{l} / \mathrm{F}_{p}
$$

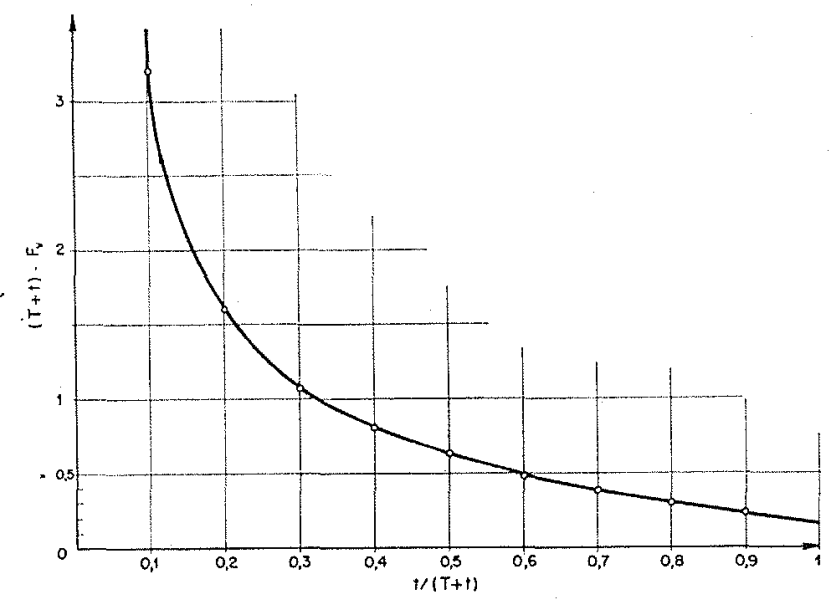

Fig. 13. - Courbe représentative de la fonction $2 \sin \left[(\pi t) /\left(\mathrm{T}+{ }^{\prime} t\right)\right]$

(6) Dacklek, loc. vit., p. 69, éq. 49.

(7) Hofmane, Voir remarque 3 , éq. 10. 
Suivant (22), il en résulte, pour la longueur iquivalente :

$$
l=\left(1+\sqrt[3]{ } t_{1} / t_{2}\right) 2\left(\mathrm{~T}+t_{2}\right) \sin \left|\frac{45 t_{2}}{\mathrm{~T}+t_{2}}\right|^{\prime \prime}
$$

\section{m) Application a UN MUR}

Dans le cas d'autres ouvrages on peut, de la mème façon que ci-dessus, trouver de manière approchée les fonctions $F$, $f$ et la longueur $l$ à partir du résean symétrique de l'écoulement sous un rideau de palplanches. La section la plus fréquente est le rectangle représenté sur la figure 2. Pour celui-ci, on peut décomposer l'écoulement en trois zones (fig. 14) : celles qui sont de part et d'autre du mur et correspondent aux réseaux considérés jusqu'ici, et une troisième zone située entre les deux premières et pouvant être assimilée à un réseau en damier (s). On obtient avec cette hypothèse les coefficients de forme suivants :

$F_{1}=\sqrt[3]{\mathrm{T} / t_{1}}$

$\mathrm{F}_{2}={ }^{\top} \mathrm{T} / \mathrm{P}$

(8) Voir aussi DAcHLek, op. cil., p. 76.

$$
\begin{aligned}
& \mathrm{F}_{3}=\forall / \mathrm{T} / t_{2} \\
& \mathrm{~F}=\frac{1}{\sqrt{ } t_{\mathrm{l}} / \mathrm{T}+\mathrm{B} / \mathrm{T}+\mathrm{M} / \mathrm{t} / \mathrm{T}} \\
& v=\frac{2 k h F_{v}}{\sqrt[3]{t_{1} / t_{2}}+\frac{B}{\mathrm{~T}} \sqrt[3]{\frac{T}{t_{2}}}+1} \\
& l=\left(1+\sqrt[3]{t_{1} / t_{2}}+\frac{B}{\mathrm{~T}} \sqrt[3]{t_{2}^{\mathrm{T}}}\right) 2\left(\mathrm{~T}+t_{2}\right) \sin \left[\frac{4 \mathrm{a} t_{2}}{\mathrm{~T}+t_{2-}}\right]
\end{aligned}
$$

\section{n) Autres applications}

D'aulres facteurs de forme sont encore indiqués dans l'ouvrage de DAchorr. Ainsi, par exemple, pour l'écoulement symétrique sous un rideau de palplanches avec $\mathrm{T}=\infty$, on $\mathrm{a}$ :

$$
\begin{gathered}
\mathrm{F}=\frac{l}{\pi} \ln \left(\frac{z}{t}+\sqrt{\left.\left(\frac{z}{l}\right)^{2}+1\right)}\right. \\
f(z)=\frac{1}{\pi t \sqrt{1+\left(\frac{z}{t}\right)^{2}}}
\end{gathered}
$$

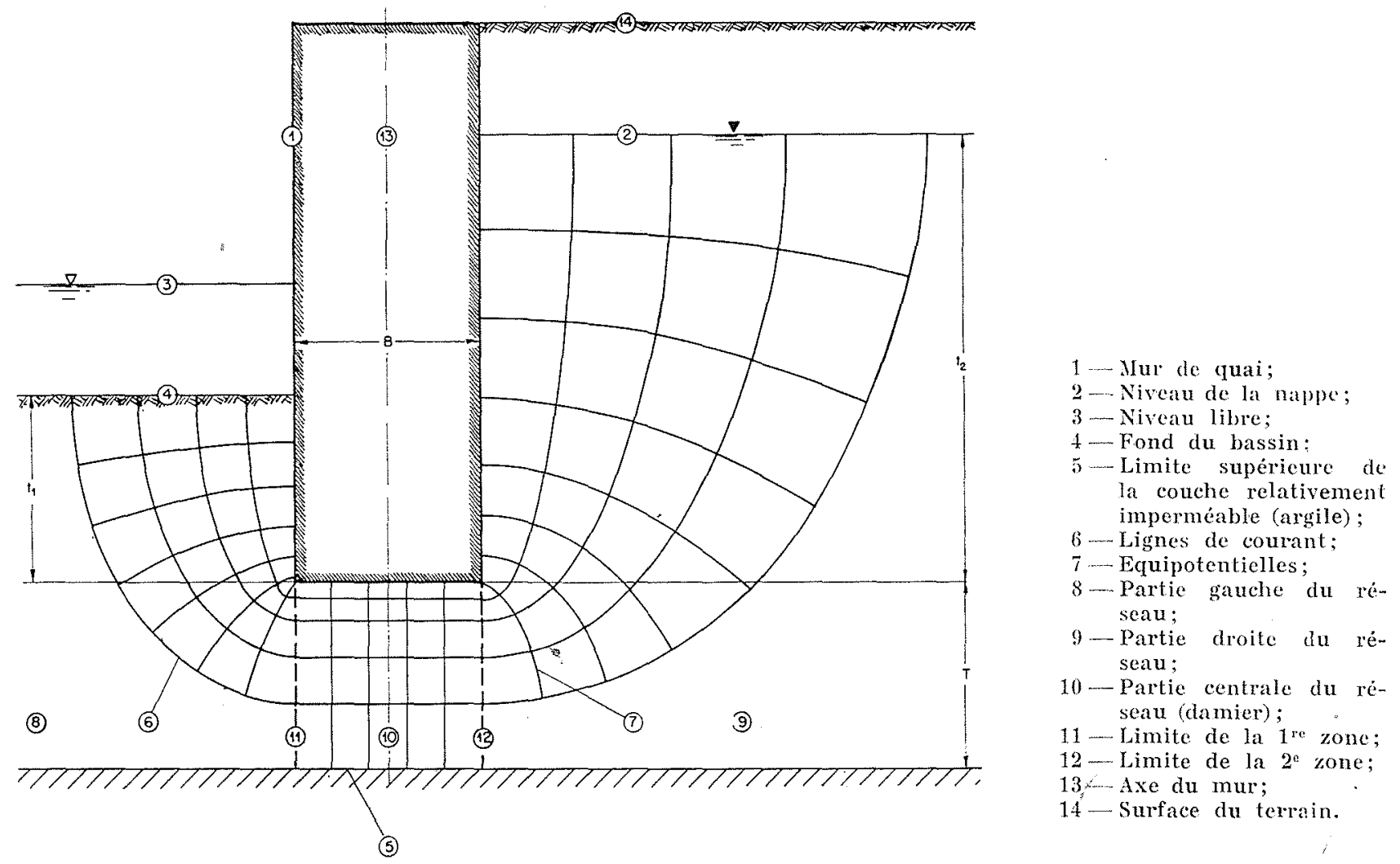

Fin. 11. -...- Tracé simplifié d'un ressau sous un mut de quai. 
Les deux fonctions dépendent de la longueur : pour laquelle on cherche le débit $q$.

Pour l'écoulement derrière un rideau de palplanches comme celui de la figure 6 (réseau divisé en deux) :

$$
\mathrm{F}=\frac{\pi}{2 \ln \sin \frac{\pi}{2} \mathrm{~T}+t}
$$

On trouve également chez DAchlir (9) la mé- thode de calcul des massifs de fondations complexes suivant cette méthode :

$$
\begin{aligned}
\Delta h_{i} & =\mathrm{A}_{i} \frac{q}{l}=h_{i}=\left(\begin{array}{c}
1 \\
\mathrm{~F}_{i}
\end{array}\right) \frac{q}{k} \\
q & =k h \quad \frac{1}{(1 / \mathrm{F})}=k h \frac{1}{\mathrm{~B}} \\
\text { on trouve } & : \\
l & =\frac{(1 / \mathrm{F})}{2\left(1 / \mathrm{F}_{p}\right) \mathrm{F}_{v}}=\frac{\mathrm{B}}{2 \mathrm{~A}_{p} \mathrm{~F}_{v}}
\end{aligned}
$$

\section{III. - SURPRESSIONS DUES A LA MARÉE}

\section{a) INTÉGRATION DE L'ÉQUATION FONDAMENTAL: SIMPLIFIÉE.}

Pour le cas particuilier où les fluctuations du niveau libre correspondent à la marée, celle-ci peut ètre exprimée de manière approchée par la fonction sinusoïdale suivante :

$$
y_{0}=a_{0} \cos n t
$$

où :

$a_{0}$ est l'amplitude de la courbe sinusoïdale ou la demi-amplitude en $m$ de la marée,

$n$ est la «pulsation » de la marée en général égale à $1,405.10^{-t}$ (en radians/s).

En introduisant lexpression (37) dans l'equation (12), on obtient :

$$
y=\mathrm{C} e^{-a t}+\alpha e^{-a t} \int a_{0} \cos n t e^{a t} d t
$$

Par suite des fluctuations périodiques du niveau libre, la nappe se trouve elle-même soumise à un mouvement résultant de la superposition de ses oscillations propres et d'une oscillation forcée en provenance de la marée et de même période que celle-ci. L'oscillation propro pour un mouvement purement périodique diminue toujours avee le temps, de sorte qu'elle peut étre négligée. Il ne reste donc pour la marée de la nappe que la deuxième partie de l'équation cidessus, laquelle, après une légère transformation, se présente finalement sous la forme suivante:

$$
\begin{array}{r}
y=a_{0} \cos [\operatorname{arctg}(n / x)] \cos [n t-\operatorname{arctg}(n / \alpha)] \\
=a_{1} \cos (n t-0)(39 a)
\end{array}
$$

en désignant par :

$a_{1}$ : la demi-amplitude de la marée de la nappe.

$\because$ : son déphasage par rapport à la marée libre.

Par conséquent, la nappe est elle aussi le siège d'une marée sinusoïdale déphasée d'un angle $q$ et dont l'amplitude $a_{1}$ n'est qu'une fraction de l'amplitude de la marée initiale. Si l'on veut trouver le temps en minutes qui correspond à l'angle exprimé en grades ou en radians, on obtient, en tenant compte du facteur $n$, les expressions suivantes :

$$
t(\min )=\frac{10.000 \pi}{1,405.60 .180}=2,07 \varphi^{0}
$$

oul :

$$
t(\min )=\frac{10.000}{1,405.60}=118,8 \text { (radians) }
$$

\section{b) Surpression hydrauligue}

On trouve pour la surpression hydraulique instantanée $h$ (suivant la figure 15)"

$$
\begin{array}{r}
h=y-y_{0}=a_{1} \cos (n t-p)-a_{0} \cos n t \\
h=\sqrt{a_{0}^{2}-2 a_{0} a_{1} \cos \varphi+a_{1}^{2}} \cos \left(n t-\vartheta_{1}\right) \\
\quad=a_{0} \sin \varphi \cos \left(n t-\vartheta_{1}\right)
\end{array}
$$

le déphasage étant donné par :

$$
\begin{aligned}
\operatorname{tg} \varphi_{1}=\frac{a_{1} \sin \varphi}{a_{1} \cos \varphi a_{0}} & =\frac{i \operatorname{tg} \varphi}{1-a_{0} /\left(a_{1} \cos \varphi\right)} \\
& =\frac{\operatorname{tg} \varphi}{1-1 / \cos ^{2} i}
\end{aligned}
$$

(9) DACHLER, op. cil., pp. 76-81. 
La surpression hydraulique maximum est :

$$
h_{\max }=a_{0} \sin \varphi
$$

Le niveau libre correspondant se trouve à la cote

$$
y_{0}=a_{0} \cos ?_{1}
$$

et ceci se produit à l'instant $t=\varphi_{1} / n$.

Le plus souvent, il est intéressant de comnaitre la surpression hydraulique pour le niveau libre minimum $(n t=\pi)$.

$$
h=-a_{0} \sin \varphi \cos \varphi_{1}
$$

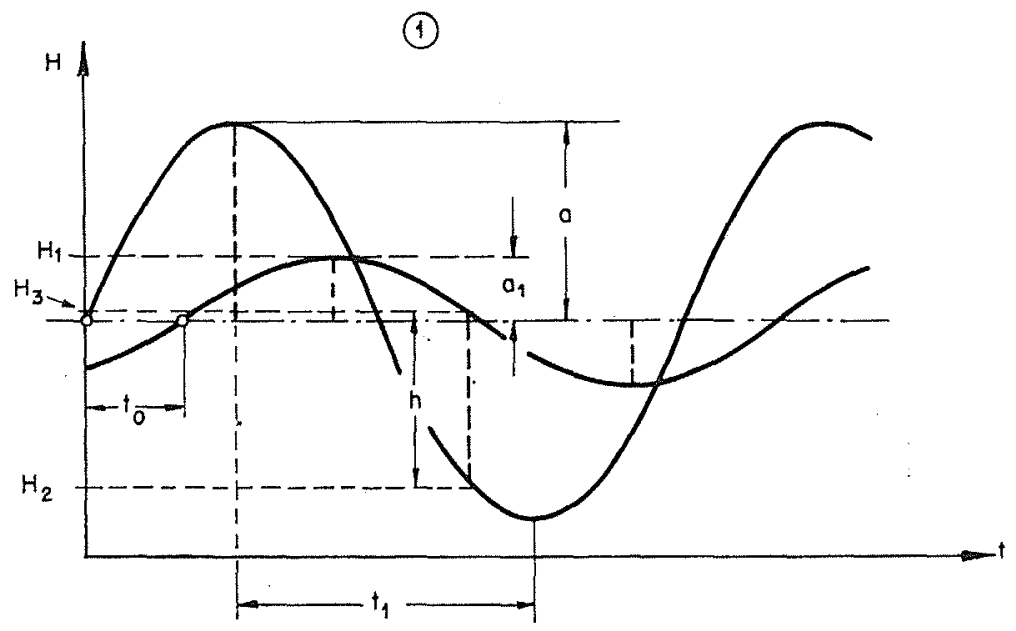

Fia. 15. - Fluctuations de la nappe derrière un mur de quai soumis à la marée.
Les équations ci-dessus ne sont valables que si les mouvements de la nappe sont exclusivement verticaux, ce qui suppose qu'il y a seulement montée et descente de l'eau derrière le mur. Ceci élimine par conséquent tout écoulement souterrain à composante principale horizontale venant du continent et se dirigeant vers le mur de quai. Dans cette hypothèse, les marées de la nappe et de la mer ont le même niveau origine.

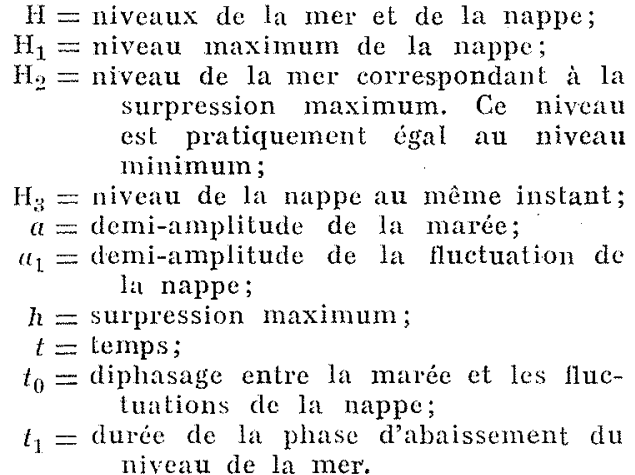
niveau de la mer.

\section{c) INTÉGRATION DE L'ÉQUATION COMPLÈTE}

Afin de contrôler la validité de l'équation simplifiée, nous nous proposons d'examiner la solution de l'équation complète. On obtient alors (10) une expression de la forme :

$$
y=y_{1}+y_{2}
$$

dans laquelle :

1) $y_{1}$ représente une oscillation propre, fonction de $t$, ayant la forme suivante :

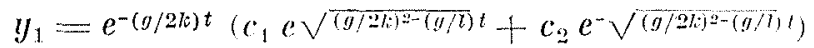

Cette expression disparaît au bout d'un temps suffisamment long. On admet alors que les con-

(10) L'établissement de l'équation complète a été déja publiée dans le mémoire de Schultze (E.), Die Grundwasserbewegung im Tidegebiet, Bautechnik, 20 (1942), pp. 111 et 112, éq. 36 (dans ce mémoire). L'éq. $36 a$ indiquée contient une erreur d'impression. La deuxième partie doit s'écrire:

$$
\cos \left(n t-\arccos \frac{1}{\sqrt{1+(n L / k \sin \gamma)^{2}}}\right)
$$

ditions d'écoulement ont atteint un état d'équilibre, et l'on néglige ce terme;

2) $y_{2}$ est une oscillation forcée de la forme :

$$
\begin{aligned}
& y_{2}=\frac{g a_{0}}{\sqrt[l]{\left(\frac{g}{l}-n^{2}\right)^{2}+\left(\frac{g n}{l}\right)^{2}}} \\
& \cdot \cos \left[n l-\operatorname{arctg} \frac{g n}{k\left(\frac{g}{l}-n^{2}\right)}\right]
\end{aligned}
$$

Pour des longueurs $l$ inférienres à $1.000 \mathrm{~m}$, $n^{2}$ est négligeable devant $g / l$.

On obtient alors :

$$
y_{2}=a_{1} \cos (n t-?)
$$

avec :

$$
\left.\operatorname{tg} \varphi=\frac{\ln }{k}=\frac{n}{\alpha} \quad \text { (en radians }\right)
$$

et :

$$
a_{1}=a_{0} \cos \varphi
$$

L'équation $(39 b)$ est identique à la solution de l'ćquation simplifiée (39 $a$ ). Dans les conditions 
envisagées, le terme $d v / d t$ s'avère numériquement négligeable et l'on peut renoncer à l'emploi de l'équation complète.

Une autre question est de savoir si, dans l'emploi du réseau isotherme, on ne devrait pas tenir encore compte du terme $v . d v / d x$ négligé jusqu'ici dans l'équation 1. Le fait est toutefois que, jusqu'à présent, ce terme était négligé dans toutes les applications des réseaux isothermes aux écoulements en milieu poreux; même dans le cas des écoulements libres (11), ce terme est considéré comme négligeable en raison de sa valeur relativement faible : cette simplification paraît done bien admissible dans le cas qui nous préoccupe.

\section{d) Exemples}

Ce sont quelques exemples qui montreront le mieux les modalités pratiques d'application de l'équation (39).

Supposons que l'on cherche à déterminer les fluctuations de la nappe et la valeur maximum de la surpression dans le cas du mur de quai de la figure 16, en supposant pour commencer que les couches (8) et (9) ont même permóabilité $\left(k=10^{-2} \mathrm{~m} / \mathrm{s}\right)$, puis, en admettant que la cou-

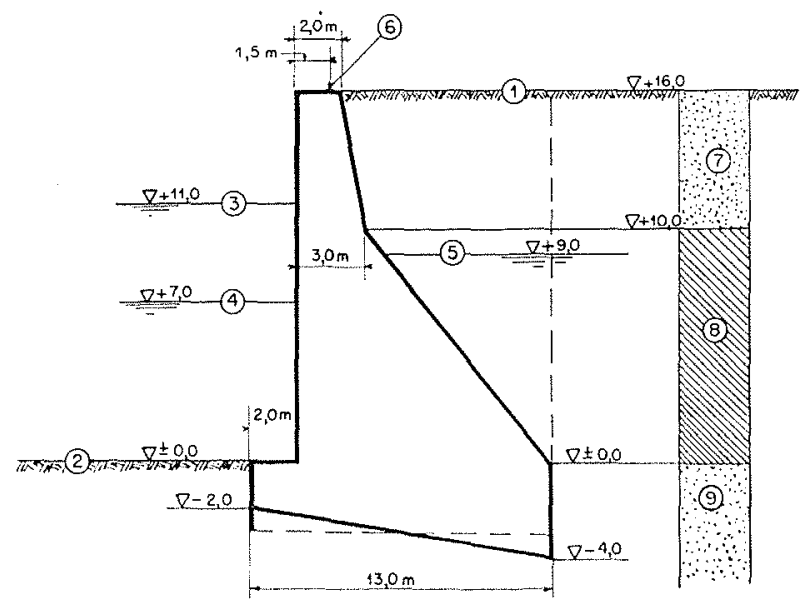

Fri. 16. - Exemple de calcul de surpresșion: coupe de l'oubrage studié.

1 -.- Surface du terrain;

2-Fond du bassin;

3 - Marée hante moyenne;

4 - Niveau de la plus basse iner;

5 - Niveau de la nappe correspondant à la plus basse mer;

6 - Rail du pont roulant;

7 -Sol sablonneux;

8-Sol argileux;

9 - Sol sablonneux.

(11) Voir Schultze (E.). - Die Berechnang des ciezeiten in Flussmilndungen, Bantechnik, 19 (1941), p. 135 . che (8) a une perméabilité $k_{1}=10^{-8} \mathrm{~m} / \mathrm{s}$ et la couche (9) une perméabilité $k_{2}=10^{-3} \mathrm{~m} / \mathrm{s}$.

D'après la figure 16 , le périmètre souterrain à prendre en considération pour la longueur du tuyau (représentation simplifiée) est le suivant: $l=2,0+$ env. $13,0+4,0+$ env. $11,0=$ env. $30,0 \mathrm{~m}$,

se décomposant en :

$$
\begin{aligned}
& \left.l_{1}=\text { env. } 11,0 \mathrm{~m} \text { [dans la couche }(8)\right], \text { et } \\
& \left.l_{9}=\text { env. } 19,0 \mathrm{~m} \text { [dans la couche }(9)\right] .
\end{aligned}
$$

L'amplitude moyenne de la marée est $2 a$ $=2,20 \mathrm{~m}$ (niv. min. moy. $=+8,80$ ). Supposons que l'écart entre la plus haute mer et la plus basse mer (ces niveaux ne correspondent pas nécessairement à une même marée) atteigne $+11,0-7,0=4.0 \mathrm{~m}$. Pour la détermination de $l$, on a admis que le niveau de la nappe correspondait à la cote $+9,0$, donc au milieu de l'écart précédent.

Dans le premier cas (perméabilité homogène), on $a$ :

$$
\alpha=10^{-2} / 30=0,033 \cdot 10^{-2}(1 / s)
$$

$$
\begin{aligned}
\operatorname{tg} \varphi & =\frac{1,405 \cdot 10^{-1}}{3,333 \cdot 10^{-4}}=0,422 \\
\varphi & =22,8^{\circ} \quad \cos \varphi=0,923 \quad \text { sin } \varphi=0,388 \\
t & =2,07 \cdot 22,8=47 \text { minutes de retard }(40 a) \\
a_{1} & =1,10 \cdot 0,923 \\
a_{1} & =1,015 \mathrm{~m}
\end{aligned}
$$$$
h_{\mathrm{11}: \mathrm{x}}=0,388 \cdot 1,10=0,426
$$

Ceci correspond à un niveau libre que l'on diduit de :

$$
\begin{gathered}
\operatorname{tg} \varphi_{1}=\frac{0,422}{1-\frac{1 / 0,923^{2}}{1-1,174}}=\frac{0,422}{1-2,42} \\
\varphi_{1}=-67,55^{\circ} \\
\cos \varphi_{1}=0,382
\end{gathered}
$$

soit :

$$
y_{0}=1,10.0,382=0,42 \mathrm{~m}
$$

Pour le niveau libre minimum, la surpression est :

$$
h=-0,426.0,382 \stackrel{!}{=}-0,16 \mathrm{~m}
$$

Par conséquent, la pression est dirigée de l'extérieur vers l'intérieur. 
Dans le second cas (perméabilité differente), on trouve:

$$
\begin{aligned}
& x_{1}=10^{-5} / 11=0,091 \cdot 10^{-5}(1 / s) \\
& \sigma_{0}=10^{-3} / 19=0,053 \cdot 10^{-3}(1 / s) \\
& \alpha=\frac{1}{\frac{11}{10^{-8}}+\frac{19}{10^{-3}}}=\frac{10^{-3}}{1.100 .000+19} \\
& x^{2}=0,091 \cdot 10^{-5}=x_{1} \\
& \operatorname{tg} \hat{i}=\frac{1,405 \cdot 10^{-4}}{0,091 \cdot 10^{-8}}=15,4 \cdot 10^{t} \\
& \text { ? } 90^{\circ} \\
& \cos \because \# 0 \\
& \sin \vartheta \# 1,000 \\
& t=2,07.89,6=186 \text { minutes de relard } \\
& a_{1} \# 0
\end{aligned}
$$

$h_{\text {max }}=1,10 \cdot 1,000 \mathrm{~m}$ aux hautes eaux

\section{e) Conchusions}

Ainsi le niveau de la nappe se trouve sans oscillations à une cote moyenne

$$
8,80+1,10=9,90 \mathrm{~m}
$$

de sorte que la surpression hydraulique serait de $2,90 \mathrm{~m}$ pour le niveau libre minimum minimorum. Par conséquent, il ressort de l'application de l'équation (39) qu'un calcul précis des oscillations $a_{1}$ de la nappe devient superflu dans le cas des sols peu perméables. Par conséquenl, si le sol est argileux et si l'on se place dans les hypothèses énoncées, on peut admettre que la nappe est à la cote correspondant au niveau libre maximum. L'équation (39) ne sera employée que s'il s'agit d'ourrages qui reposent sur un sol relativement perméable. Avec une longueur $l=$ env. $35 \mathrm{~m}$, qui correspond à une. moyenne courante pour les murs de quai, l'anplitude de la marée de la nappe, rapportée à celle du niveau libre, a pour valeurs, suivant les perméabilités :

$\begin{array}{cc}k(\mathrm{~m} / \mathrm{s}) & 100 a_{1} / a_{0} \\ 10^{-2} & 90 \\ 10^{-3} & 20 \\ 10^{-1} & 2\end{array}$

Avee des sols fortement perméables, dont la limite supérieurc correspond à environ $10^{-2} \mathrm{~m} / \mathrm{s}$, la nappe a presque le mème mouvemenl que la mer. Dès que l'on a affaire à des sols de perméabilité moyenne $\left(k=10^{-1} \mathrm{~m} / \mathrm{s}\right)$, le mouvement de marée de la nappe cesse presque complètement. Par conséquent, il n'y a que peu de types de sols dans lesquels on peut prévoir des variations périodiques du niveau de la nappe. Moins le sol est perméable, plus est grand le róle que joue le niveau moyen de la nappe qui ne peut ètre pris égal au niveau moyen libre quen l'absence de toute alimentation extérieure de la nappe. Dans le cas de plusieurs couches de perméabilité différente, il se forme dans la nappe plusieurs étages dont les oscillations sont soumises à des influences do diverses natures. En outre, à une stratification horizontale du sol correspond le plus souvent un cicoulement horizontal pour lequel les calculs précédents ne sont plus valables.

\section{f) Calcul précis de l'exemple}

Un calcul précis de la longueur $l$ pour un réseau isotherme doit avoir comme point de départ une section simplifiée du mur avec surface arrière verticale. Par conséquent, si l'on trace sur la figure 16 une verticale passant par l'arèle ar. rière de lonvage, on obtient comme périmetre souterrain :

$$
l=3+13+3+9=28 \mathrm{~m}
$$

Si l'on se donne :

$$
\mathrm{T}=20 \mathrm{~m}, t_{1}=3 \mathrm{~m}, t_{2}=12 \mathrm{~m}
$$

le calcul précis conduit à :

$$
\begin{aligned}
l= & \left(1+\sqrt[3]{3 / 12}+\frac{13}{20 \sqrt[3]{12 / 20}}\right) 2(20+12) \sin \frac{12}{4.32} \\
& =(1+0,63+0,77) 64 \sin 16,85 "=44,5 \mathrm{~m}
\end{aligned}
$$

Si on prend $\mathrm{I}=10 \mathrm{~m}$, on trouve :

$$
\begin{aligned}
l & =\left(1+0,63+\frac{13}{10 \sqrt[3]{1,2}}\right) 2,22 \sin \frac{12 \pi}{4,22} \\
& =(1+0,63+1,22) 44 \sin 24,5^{\circ}=52,0 \mathrm{~m}
\end{aligned}
$$

Le calcul précis donne donc des valeurs presque doubles de celles du calcul approché. Pour des sols ayant un $k$ compris entre $10^{-2}$ et $10^{-1} \mathrm{~m} / \mathrm{s}$, le calcul précis s'impose afin de ne pas obtenir des valeurs erronées pour l'amplitude des fluctuations de la nappe. De plus, la hauteur de la couche perméable exerce une influence.

Mais, dans l'ensemble, on peut ne pas attribuer une trop grande importance aux crreurs dues à la 


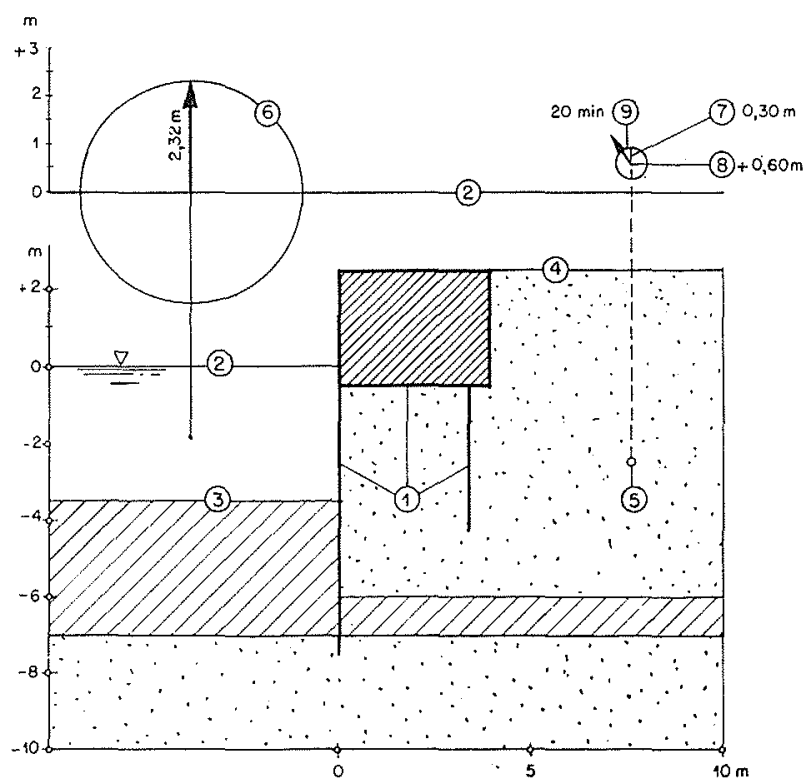

Fua. 17. - Fluctuations de pression dans la nappe derriere un quai fondé sur pieux à Emden (Baut. 10) (1932), p. 945.

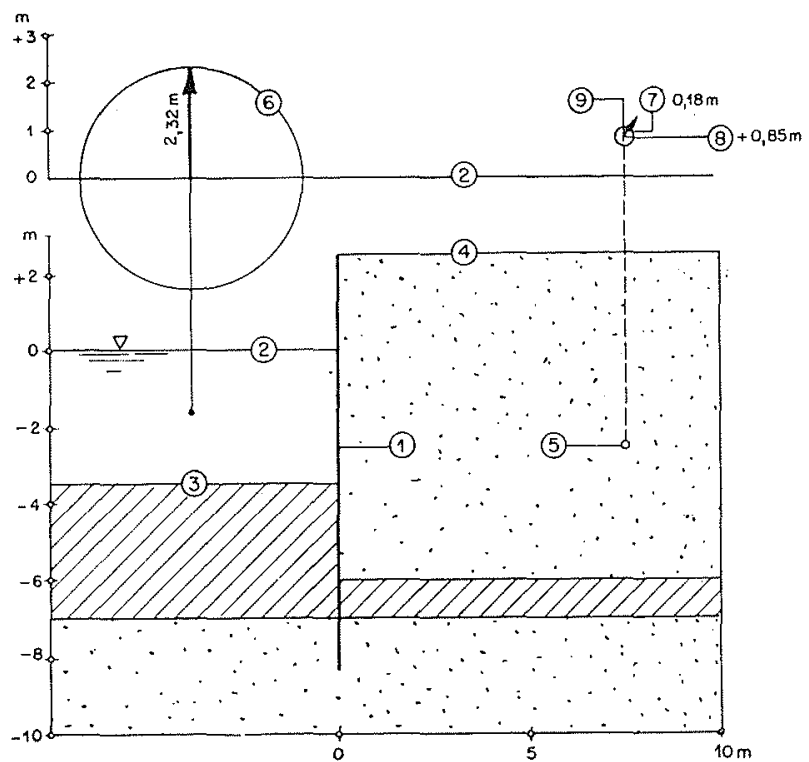

Fra. 18. - Flactuations de pression dans la nappe derriere un rideau de palplanches it Emden (Baut. 11) (1933), p. 666.

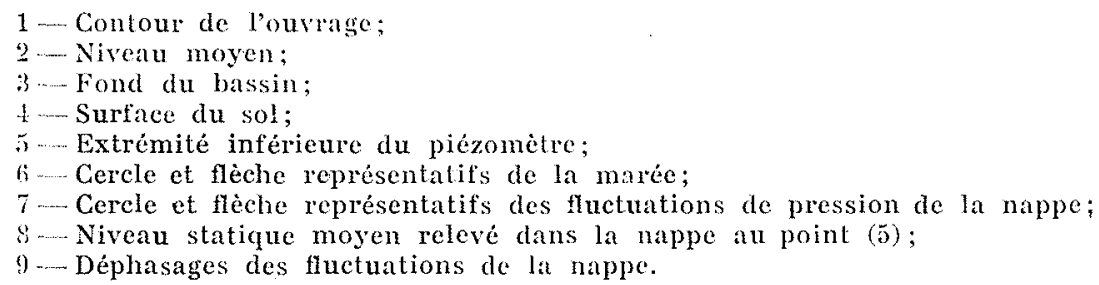

détermination approchéc de la longueur $l$, car de faibles irrégularités dans le sol, qui suffisent le plus souvent à provoquer des écarts considérables de la perméabilité $k$, ont des répercussions beaucoup plus importantes.

\section{g) Comparaison avec les observations}

Afin de se rendre compte d'une manière générale dans quelle mesure les hypothèses admises dans l'établissement des équations précédentes concordent avec la réalité, on a résumé de manière schématique sur la figure 17 quelques observations faites immédiatement derrière les murs de quai dans une région à marée. L'amplilude de la marée du niveau libre correspond à la longueur de la grande flèche qui est située en haut et à gauche de la figure et qui est dirigée verticalement vers le haut dans un cercle (6). Les marées dans la nappe sont également représentées par des cercles (9) dont le diamètre correspond à l'amplitude et dans lesquels sont placées des flèches orientées de façon à indiquer le retard ou l'avance de la marée souterraine par rapport à la marée libre. Par exemple sur la figure 17 , alors que la flèche-repère de la marée libre marque 12 heures, la flèche de la marée souterraine se trouve du còté de 11 heures, indiquant que la nappe atteint son niveau maximum 20 minutes après la haute mer (9). De plus, le schéma montre que la demi-amplitude de la marée (7) de la nappe est $0,30 \mathrm{~m}$, tandis que celle de la marée extérieure (6) atteint $2,32 \mathrm{~m}$. De plus, on voit que le niveau moyen de la nappe (8) est à $0,60 \mathrm{~m}$ au-dessus de celui de la mer (2). La figure 18 , de son còté, fait ressortir un niveau moyen de la marée souterraine (8) supérieur de $0,85 \mathrm{~m}$ à celui de la mer (2). On a représenté sur la figure 21 les diverses marées décelées au sein de la nappe dans le cas d'un terrain stratifié. Les points de la nappe ayant fait l'objet d'un sondage sont identifiés par des chiffres romains. A $25 \mathrm{~m}$ du mur, on trouve déjà pour la pression moyenne des écarts, avec le niveau moyen de la mer, allant jusqu'à $1,44 \mathrm{~m}$. Cependant, au voisinage du mur de quai (à; une distance de $10-15 \mathrm{~m}$ ) la pression moyenne se trouve varier entre $+0,35$ (fig. 21) et $+3,50 \mathrm{~m}$ (fig. 22) pour la couche supérieure, et entre - 0,68 (fig. 21) et $+0,62 \mathrm{~m}$ (fig. 19, ici à une distance de $30 \mathrm{~m}$ ) 


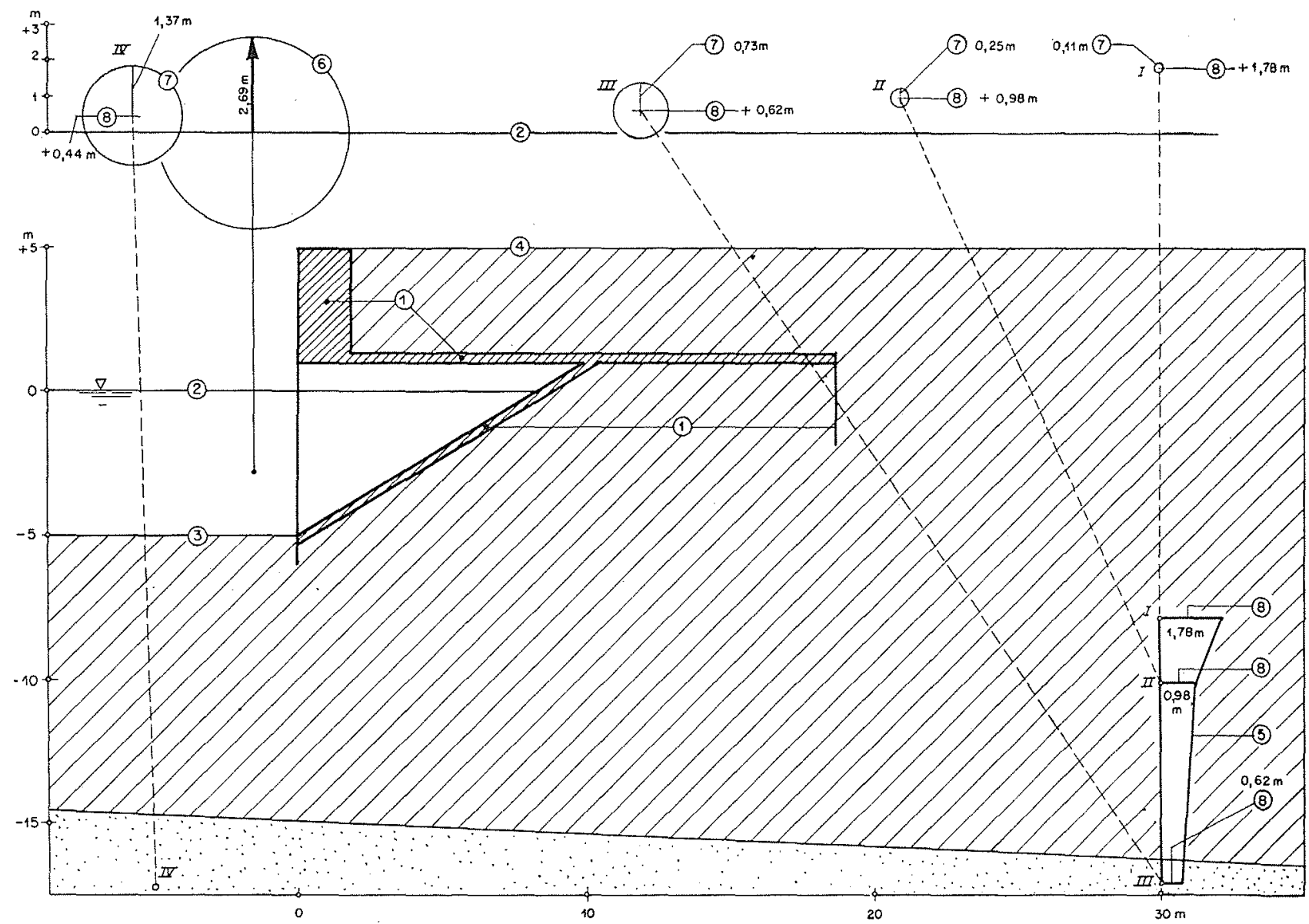

Frg. 19. - Fluctuations de pression dans la nappe derrière un mur quat à Emden

1 - Contour de l'ouvrage;

(Baut. 12) (1934), p. 597.

2 - Niveau moyen;

3 - Fond du bassin;

4 - Surface du sol;

5 - Variation des pressions moyennes le long de la verticale I-III;

6-Cercle et flèche représentatifs de la marée;

7 - Cercle et flèche représentatifs des fluctuations de pression de la nappe;

8 - Niveaux statiques moyens relevés dans la nappe et rapportés au niveau moyen de Ia mer;

I, II, III, IV. - Extrémités inférieures des piézomètres.

pour la couche inférieure. Dans l'argile, les mesures ont donné de $0,98 \mathrm{~m}$ à $1,78 \mathrm{~m}$ (fig. 19). Les différences locales sont parfois considérables (fig. 19 et 21). L'hypothèse que le niveau moyen est le même pour la marée souterraine et pour la marée libre ne se trouve pas vérifiée dans la plupart des cas observés. Par conséquent, il faut admettre que la région continentale ešt toujoursdrainée vers le mur. Le plan d'eau derrière le mur subit, de ce fait, une remontée. En outre, les oscillations propres, négligées dans un mouvement purement périodique, peuvent par contre jouer un certain rôle dans les fluctuations irrégulières de la réalité. Il faut ajouter à cela que, dans les conditions de stratification compliquées des figures 16 à 23 , et par le jeu des couches imperméables pratiquement assimilables à des parois quasi étanches, l'écoulement est dirigé suivant des trajectoires fixes et le plus souvent horizontales. L'hypothèse que l'écoulement souterrain qui contourne le mur se fait surtout en direction verticale, n'est alors plus vérifiée. Par conséquent, avant d'appliquer les équations établies, il faut s'assurer dans chaque cas particulier que les hypothèses, avec lesquelles elles ont été établies, correspondent à la réalité.

On peut déduire, en particulier, des figures les remarques suivantes :

Dans le cas de la figure 17, la couche imperméable rend difficile l'égalisation des pressions dans la nappe et dans la mer : $\cos \varphi=a_{1} / \alpha=0,30 / 2,32=0,13$, soit $: \varphi=82,5^{\circ}$. 
Par contre, le retard de vingt minutes conduit à $\varphi=20 / 2,07=9,7^{\circ}$. Il ne concorde pas avec le chiffre précédent. L'élévation de la pression moyenne semble traduire une alimentation de la nappe depuis le continent. On peut supposer que les divers niveaux moyens sont alignés suivant une droite de pente $0,60 / 7,5=0,08$.

La figure 18 correspond à des conditions analogues à celles de la figure 17. Le déphasage a changé de signe et la marée souterraine prend les devants, - fait pour lequel aucune explication plausible n'a pu être trouvée jusqu'à présent. Les niveaux moyens s'aligneraient suivant une pente $0,85 / 7,50=\mathbf{0 , 1 1}$.

Dans le cas de la figure 19 , les mesures portaient sur deux couches. La pente du niveau moyen diminue de haut en bas. La couche argileuse est drainée par la couche de sable sous-jacente. La nappe et la mer doivent communiquer quelque part, sans quoi l'amortissement devrait être plus grand (dans le cas de IV en particulier) que $1,37 / 2,69=0,51=\cos \varphi$. L'amortissement croissant de III à $I$ indique une pénétration des marées par le bas. L'écoulement était probablement vertical.

Dans la figure 20, l'eau souterraine s'écoule de la couche intermédiaire aussi bien vers le haut que vers le bas, comme il ressort des pentes II-I et II-III. Le drainage est de ce fait plus fort vers le bas. On déduit de l'amorlissement :

pour le point I :

$$
\cos \varphi=4,3 / 5,25=0,82, \text { soit } \varphi=35^{\circ}
$$

pour le point II :

$$
\cos \varphi=3,33 / 5,25=0,63, \text { soit } \varphi=50,7^{\circ}
$$

pour le point III :

$$
\cos \varphi=2,22 / 5,25=0,42, \text { soit } \varphi=65^{\circ}
$$

1 - Contour de l'ouvrage;

2 - Niveau moyen;

3 - Fond du bassin;

4-Surface du sol;

5 -Variation des pressions moyennes le long de la verticale I-III;

6-Cercle et flèche représentatifs de la marée;

7 -Cercle et flèche représentatifs des fluctuations de pression de la nappe;

$8-\mathrm{Niveaux}$ statiques moyens relevés dans la nappe et rapportés au niveau moyen de la mer $=$ surpressions moyennes dues à la nappe ;

9 - Déphasages des fluctuations de la nappe;

I, II, III. - Extrémités inférieures des piézomètres.

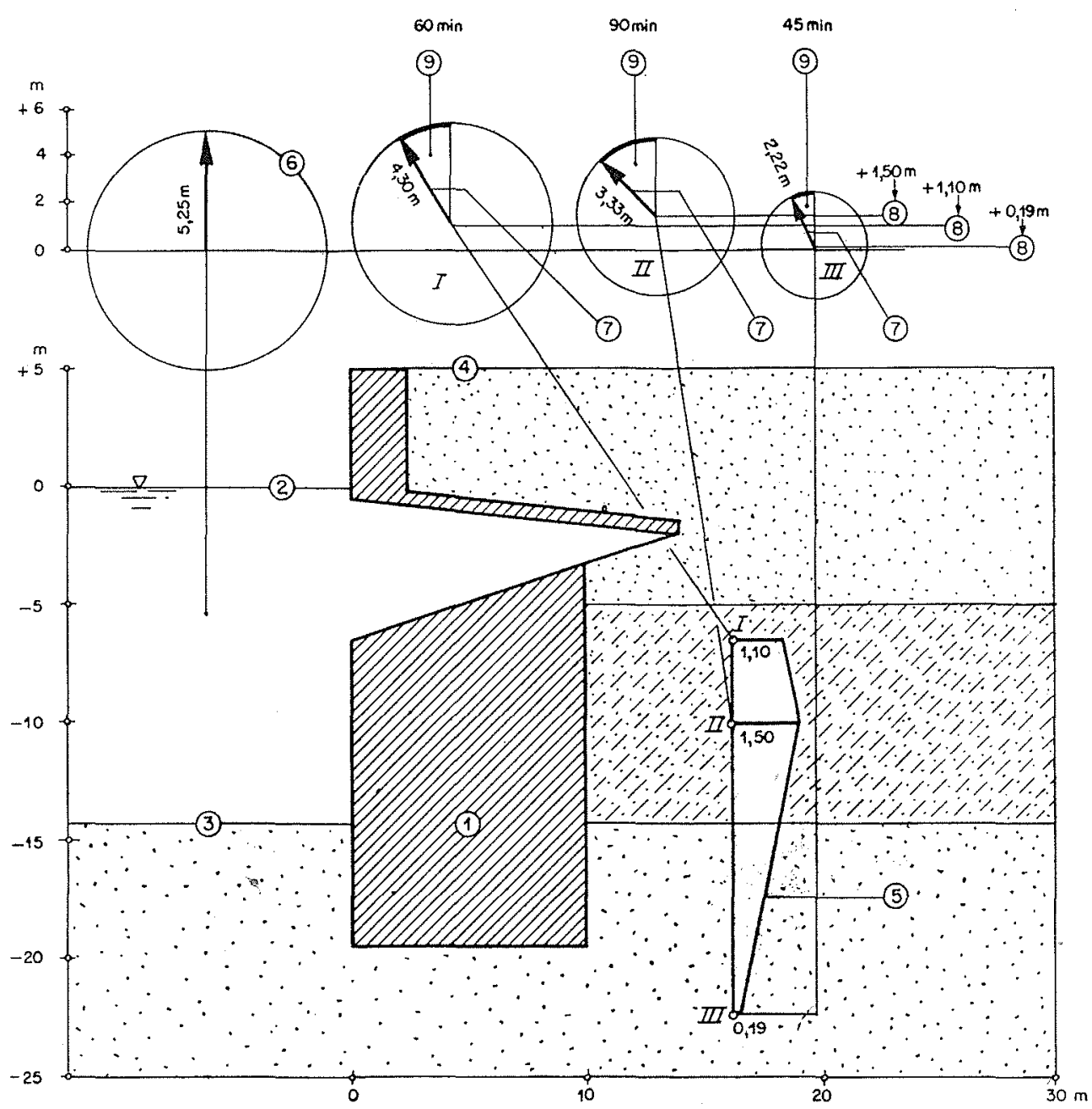

Fia. 20. - Fluctuations de pression dans la nappe derrière un mur de quai au Havre. (Congrès International de la Navigation, Bruxelles, 1935, rapport $\mathrm{n}^{\circ}$ 109.) 
Par contre, les déphasages des marées souterraines conduisent aux angles suivants :

$$
\begin{aligned}
& \text { pour le point } I \quad: \$=60 / 2,07=29,0^{\circ} \\
& \text { pour le point II : } 0=90 / 2,07=43,5^{\circ} \\
& \text { pour le point III : }:=45 / 2,07=21,7^{\circ}
\end{aligned}
$$

Ce n'est qu'au dernier point que les valeurs de ô, déduites de l'amortissement et du dépha- sage, ne concordent pas. L’amortissement décelé de I à II et III ne peut s'expliquer qu'en admettant une communication directe entre la mer et la couche sablonneuse supérieure, communication offrant à l'eau un trajet plus court que celui qui doit contourner l'ouvrage par le bas. Il est probable d'ailleurs que les effets de l'alimentation par le haut et de l'alimentation par le bas se superposent, rendant toute vérification particulièrement difficile.

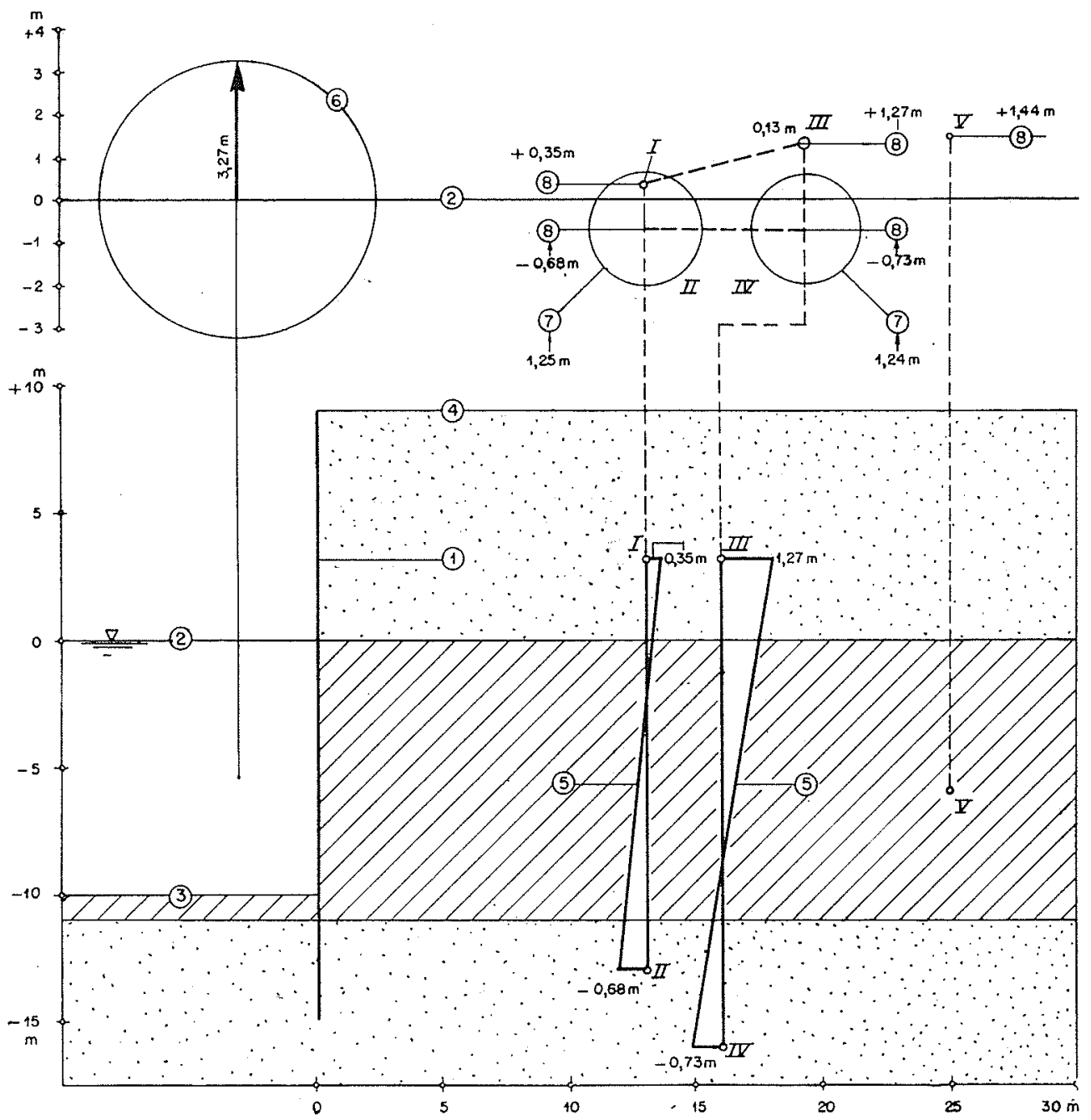

Fia, 21. - Fluctuations de pression dans la nappe derriere un mur de quai dans le port de Brème. (Agatz-Kampf des Ingénieurs, Berlin 1936, p. 58.)
1 - Contour de l'ouvrage;

2 - Niveau moyen;

3 - Fond du bassin;

4 - Surface du sol;

5 -Variations des pressions moyennes le long des verticales I, II et III, IV;

(i.-Cercle et flèche représentatifs de la marée;

7 - Cercle et flèche représentatifs des fluctuations de pression de la nappe;

8-Niveaux statiques moyens relevés dans la nappe et rapportés au niveau de la mer = surpressions moyennes dues à la nappe;

I, II, III, IV, V. - Extrémités inférieures des piézomètres.
Dans la figure 21, le fait que les miveaux moyens sont, dans la couche inférieure, en dessous du niveau moyen libre, est incompréhensible, d'autant plus que l'amplitude relativement forte de la marée souterraine $(\cos \varphi=1,25 / 3,27$ $=0,38$ en II) dénote une bonne communication avec la mer. La couche supérieure semble n'avoir à peu près aucune relation avec la marée extérieure : de plus, l'eau en provenance du conti- nent tend à s'y emmagasiner; cependant, elle est drainée vers le mur (forte pente I-III), probablement vers le bas (pentes I-II et III-IV) et probablement aussi perpendiculairement au plan de la figure. Le résultat de la mesure en $\mathrm{V}$ dans la couche argileuse concorde avec I et III.

La figure 22 confirme d'une part l'indépendance presque complète de la couche supérieure vis-à-vis de la mer, d'autre part l'existence d'une 
1-Contour de l'ouviage:

2 -.- Niveau moyen;

3 .... Fond du bassin;

+ Surface du sol;

$5-.$. Extrémité inférieure du piézomètre;

6 -...- Cercle et flèche representatifs de la marée;

8.-..- Niveau statique moyen rolevé dans la nappe au point (5);
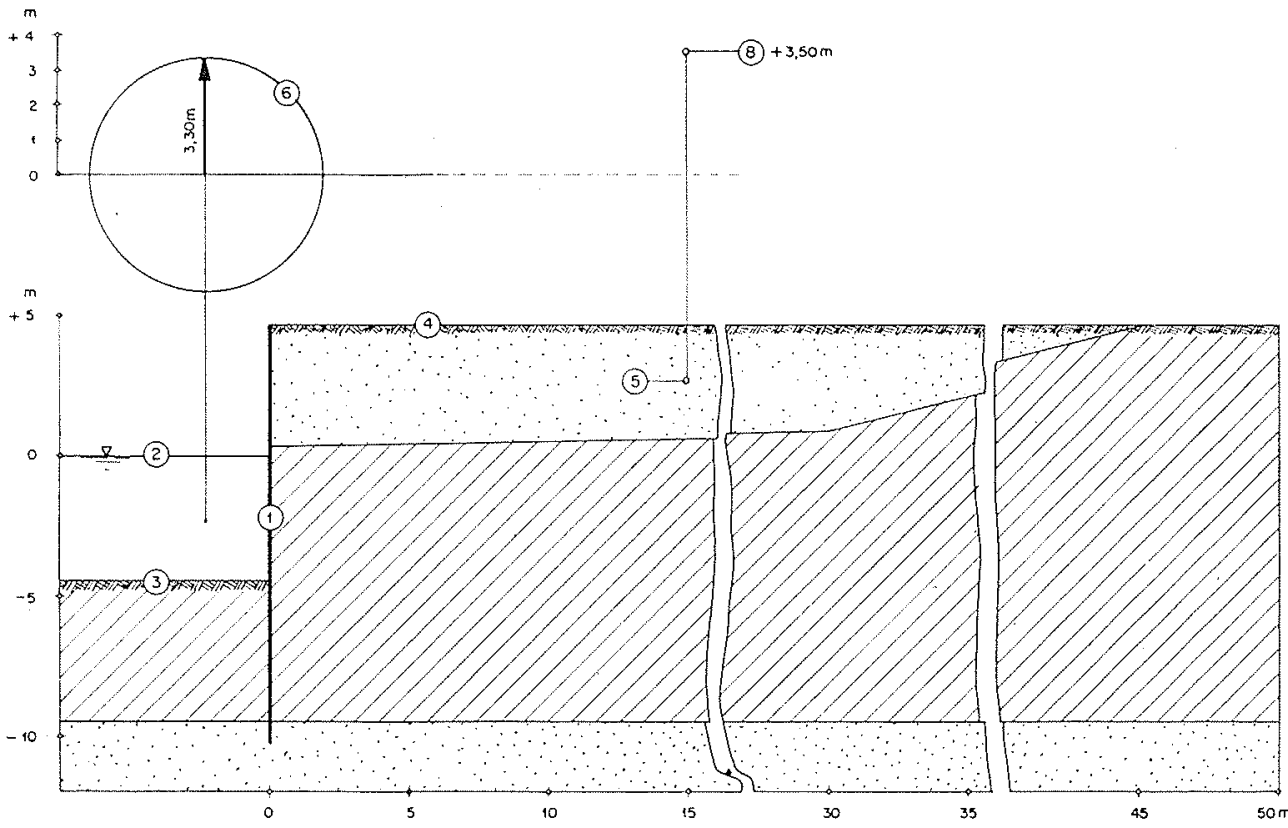

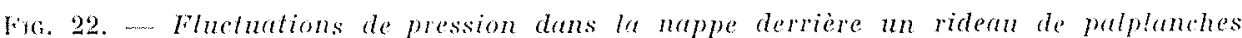
a Nordenham.

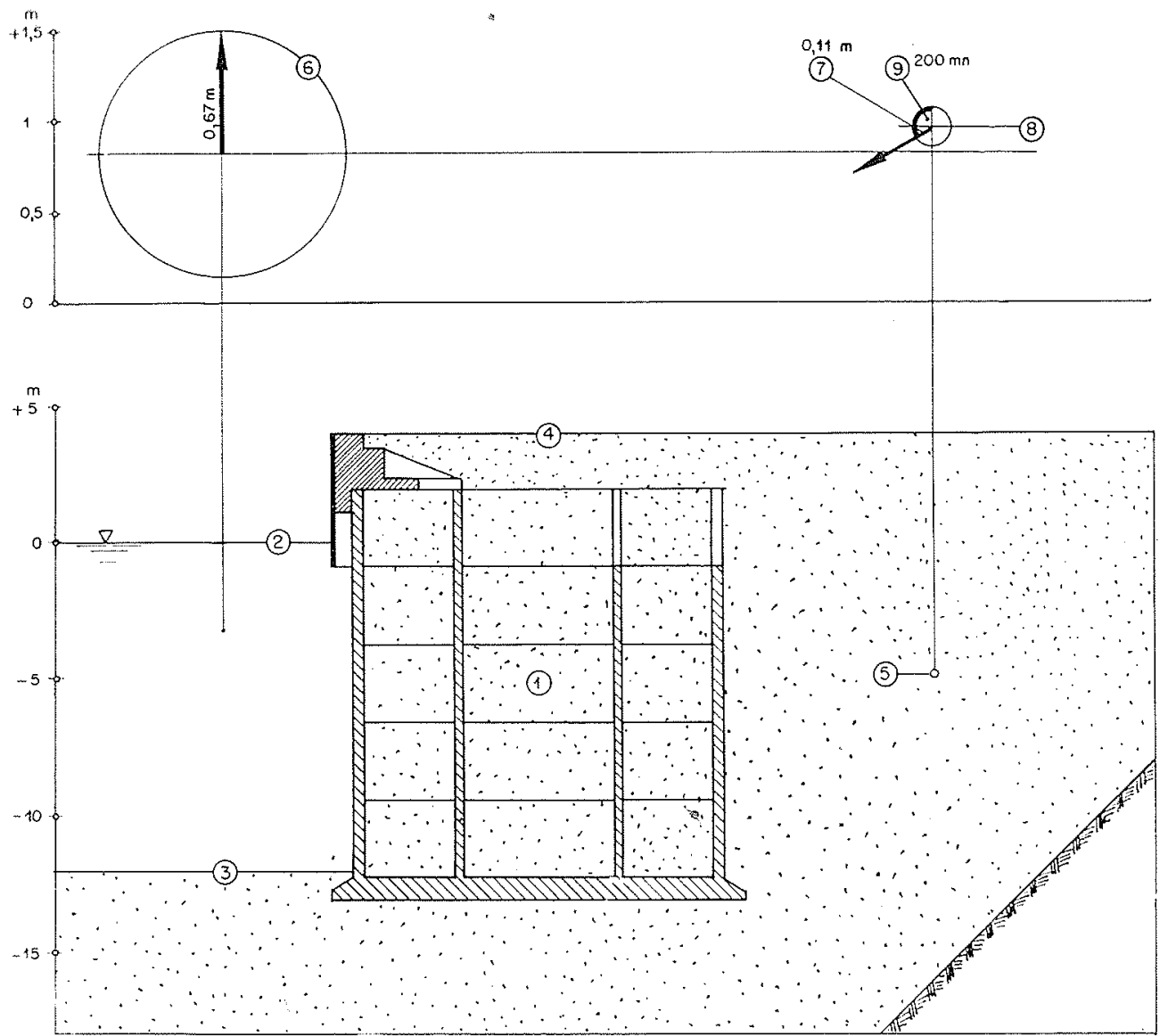

Fra. 23. - Fluctuations de pression dans la nappe derribre un mar de quat a Rotterdam. Werft. Reederei, Hafen, 17, 1936, p. 76.
1 -... Contour de l'ourrage;

2 - Niveau moyen;

3 - Fond da bassin;

4 -..- Surface du sol;

5) Extrémité inférieure du piezometre:

6-.-. Cerele et flèche représentatifs de la marée;

7 -... Cercle et fleche représentatif's des fluctuations de pression de la mappe;

- Niveau statique moyen releve dans la nappe au point (5):

9-Dóphasages des fluctuations de la nappe; 
retenue considérable derrière l'ouvage. La pente du niveau moyen est $3,50 / 15=0,23$.

Sur la figure 23 , on a représenté un cas particulierement simple dans lequel il n'existe qu'une seule couche et où la nappe souterraine a ćté sondée à une distance relativement courte de la face arrière du mur. L’angle $p$ est déterminé de la manière suivante : du rapport des amplitudes on déduit : $\cos \varphi=0,11 / 0,67=0,16$ et $\varphi=80,8^{\circ}$; du déphasage il résulte : $\varphi=$ env. $200 \mathrm{~min} / 2,07$ $=97^{\circ}$. Sans qu'elle soit parfaite, la concordance de ces deux valeurs est assez satisfaisante.

D'une facon générale, on doit dans l'appréciation des observations, tenir compte du fait que, dans certains cas, le problème comporte probablement trois dimensions puisque la nappe communique directement avec la mer par des trajets contournant le mur et ne figurant pas dans le plan de figure. De plus, certains sondages, effectués relativement loin de la face arrière du mur, ne suffisent pas nécessairement à déterminer la surpression. Les marées ne sont pas des courbes purement sinusoïdales et le niveau moyen n'est pas une constante. Cependant les mesures, peu nombreuses, montrent, même pour la figure 17 , une concordance de principe entre la théorie et la réalité. Mais tant que l'on ne disposera pas d'observations plus nombreuses, il sera impossible de dire quelque chose de plus précis sur ce sujet. Malheureusement, les observations sûres relatives à la surpression derrière un mur de quai sont encore rares jusqu'à présent. Les données dont on dispose sont done encore trop insuffisantes pour que l'on puisse en tirer des conclusions générales.

\section{h) LES LOIS}

Aussi, bien qu'on ne puisse pas s'attendre, sur le plan numérique, à une concordance parfaite avec la réalité, dès qu'on se trouve en présence de conditions de sol compliquées, l'étude des équations montre néanmoins quelle est la nature, et, dans une certaine mesure, l'influenec relative des divers facteurs déterminant la valeur des surpressions derriere un mur de quai. Ces facteurs se réduisent à $k$ et $l$. L'influence de $k$ peut se résumer ainsi : théoriquement, Iorsque le sol atteint un certain degré d'imperméabilité (correspondant à peu près à $k<10^{-2} \mathrm{~cm} / \mathrm{sec}$ ), les mouvements de la nappe disparaissent en général presque totalement. La nappe s'établit alors à un niveau moyen, de sorte que le calcul de ses marées devient superflu. Mais le niveau moyen lui-mème ne peul pas ètre calculé; il en résulte que les équations établies ne peuvent être appliquées que lorsqu'il s'agit de sols relativement perinéables.

Le facteur $l$, ou la fonction $f(z)$ qui lui correspond, dépendent de la longueur du trajet parcouru par les eaux de la nappe. Il ressort qu'un raccourcissement de ce trajet conduit à une augmentation de l'amplitude de la marée souterraine et à une réduction de son déphasage. Par conséquent, la surpression hydraulique est d'aulant plus faible que la communication entre la nappe et la mer est plus directe, et cette relation peut être considérée comme à peu près linéaire. D'après l'équation (42a), on a :

$$
h_{\max } / a_{0}=\sin p=(n / \alpha) / \sqrt{1+(n / x)^{2}} .
$$

Pour un sol perméable (où $\alpha$ est grand), seul cas pris en considération, $h_{\max } / \alpha_{0}$ tend vers $l(n / k)$ et est donc directement proportionnel à la longueur du trajet et inversement proportionnel à la perméabilité. Ceci montre bien l'intérêt des drainages qui raccourcissent considerablement le trajet des eaux souterraines. Mais il faut tenir compte du fait qu'une réduction de la perméabilité $k$, susceptible d'être provoquée par l'encrassage des drains, fait immédiatement remonter la surpression hydraulique. Etant donné que les valeurs de $l i$ et de $l$ figurent toujour's ensemble sous la forme du facteur $z$ dans les équations, mais que les variations de $k$ sont considérablement plus grandes que celles de $l$, un accroissement de la perméabilité peut causer un accroissement des trajets, même si ceux-ci sont courts.

\section{IV. - LES SURPRESSIONS SUR LES MURS DE QUAI LE LONG DES COURS D'EAU}

\section{a) Solution Approchéx:}

Etant dommé l'irrégularité des fluctuations de niveaux d'une rivière, il est difficile de tronver une fonction déterminée $y_{0}$ dont la validité ne soit pas limitée à un cas particulier. Cependant, afin d'obtenir une solution utilisable autant que possible dans tous les cas, nous adopterons, pour le niveau libre, une loi de variation particulierement défavorable, et nous chercherons à en déduire les fluctuations de la nappe. De semblables conditions se trouvent évidemment réunies lorsque le niveau de la riviére, après s'être assez longtemps maintenu à une cote (fig. 1) relativement élevée, vient à s'abaisser rapidement. Si l'on admet quo l'abaissement peut être repré- 
senté avec une précision suffisante par une droite, on obtient une loi de variation (2) du niveau de la rivière telle que celle de la figure 24 . Dans chaque cas particulier on doit évidemment ajuster le niveaux (1) et (4), ainsi que la loi (2), suivant les observations effectivement réalisées. Par conséquent, on notera soigneusement, dans les relevés hydrographiques, les sections où des étiages prononcés suivent de très près des niveaux relativement élevés.

Dans le cas de la figure 24 , on peut exprimer la loi de variation du niveau libre par la formule :

$$
y_{0}=a-b t
$$

où $b$ représente vitesse d'abaissement du niveau libre.

Cette equation est valable depuis l'instant $t=0$ jusqu'à l'instant $t=t_{1}$. Par conséquent, si l'on part de nouveau de l'équation aux différences finies (11), on peut y poser :

$$
y_{1}=y_{0}
$$

car on admet que le niveau ( $a$ ) est établi depuis un temps suffisamment long pour que la nappe s'y soit ajustée. Si l'on considère ensuite que lion peut écrire, pour la surpression hydraulique:

$$
h=y_{2}-\left(a-b t_{1}\right)
$$

on tire des équations (11), (44) et (45), pour la valeur approchée de la surpression à l'instant le plus defavorable $t_{1}$, l'expression suivante :

$$
h_{\operatorname{mix}}=\frac{b t_{1}}{1+\frac{\alpha}{2} t_{1}}
$$

Cette équation permet de calculer la valeur de la surpression, commaissant :

la durée $t_{1}$ de labaissement du niveau libre,

la vitesse d'abaissement $b$,

la perméabilite du sol $k$,

et la longueur $l$ du trajet dinfiltration contournant l'ouvrage.

Ce calcul, tres simple, permet de faire une évaluation rapide de l'ordre de grandeureprobable de la surpression. On voil que celle-ci croît proportionnellement à $b$, vitesse d'abaissement du niveau libre, et qu'elle dépend en outre tris étroitement de $k$ et de $l$.

La variation de $h$, obtenue d'après l'équation (46), est représentée par la droite (3) de la figure 24.

\section{b) INTÉgration de L'ÉQuation SIMPLIFIÉE}

L'application de la formule approchée (46) se heurte à des difficultés, lorsque l'intervalle de temps pendant lequel on admet un abaissement linéaire du niveau de la nappe devient grand. Dans ce cas, on doit recourir à la solution précise des équations (9) ou (13), telle qu'elle est donnée par les équations (12) et (15). Si l'on emploie d'abord l'équation simplifiée (12), on détermine la constante d'intégration $\mathrm{C}$ par la condition :

$$
t=0 ; \quad y=y_{0}=a
$$

On obtient alor's, d'après l'équation (12), l'expression suivante donnant la loi de variation, en fonction du temps, du niveau de la nappe :

$$
y=a-b(t-1 / x)-e^{-\alpha t} b / x
$$

Afin d'obtenir la valeur maximum de la surpression, on part à nouveau du fait que celle-ci s'établit à l'instant $t_{1}$ où l'abaissement du niveau libre atteint justement sa limite extrème :

$$
t=t_{1} ; h=h_{\operatorname{mix}}=y_{t=t_{\mathrm{r}}}-\left(a-b t_{1}\right)
$$

Si, dans l'équation (48), on introduit la valeur de $y$ tirée de l'équation (47), on obtient pour la valeur de la surpression hydraulique derrière un mur de quai l'expression plus précise suivante :

$$
h_{\max }=\frac{b}{\alpha}\left(1-e^{-\alpha t_{1}}\right)
$$

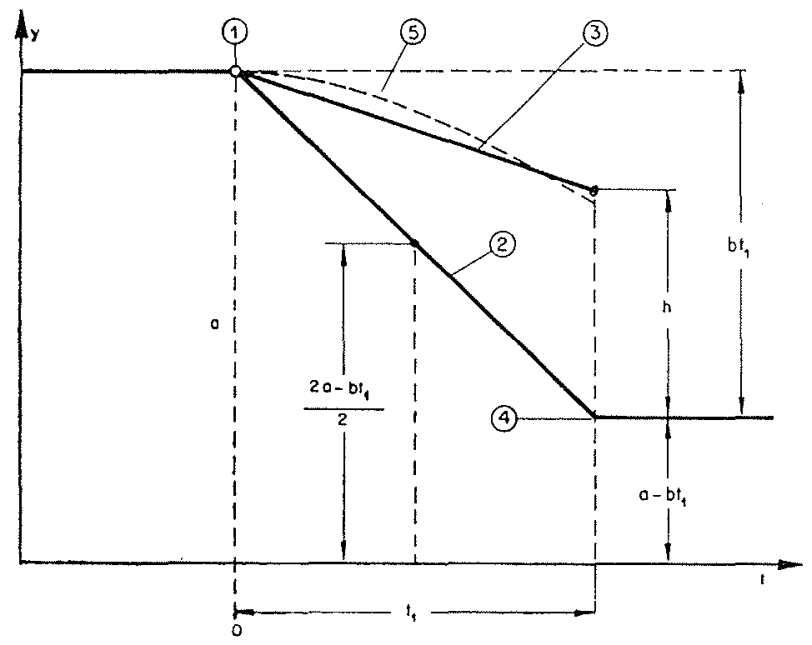

Fin. 24. -- Calcul approché des surpressions dans le cas d'un abaissement linéaire du niveau libre.

1 - Point de départ de l'abaissement;

2 -Loi connue d'abaissement du niveau libre;

3 -.- Variation correspondante de la nappe déduite du, calcul approche;

+ - Point final de l'abaissement du niveau libre;

5 -.. Variation de la nappe déduite d'un calcul plus rigoureux. 
On a porté sur la figure 24 la courbe (5) représentant la variation de la nappe telle qu'elle se déduit de l'équation (49). On voit qu'elle s'écarte par endroits notablement de la droite approchée (3), mais que le résultat final $h$, qui importe ici, est sensiblement le même pour la courbe et pour la droite. Toutefois, cela peut ne pas être toujours le cas et, par conséquent, il convient de se servir de l'équation (49) au lieu de l'équation (45), lorsque les intervalles de temps sont grands.

Quand les valeur's de $t_{1}$ sont très petites, les tables habituelles de la fonction $e^{a t}$ sont insuffisantes et il vaut mieux la transformer en une série :

$h_{\max }=\frac{b}{\alpha}\left(\alpha t_{1}-\frac{\left(\alpha t_{1}\right)^{2}}{2 !}+\frac{\left(\alpha t_{1}\right)^{3}}{3 !}-\frac{\left(\alpha t_{1}\right)^{4}}{4 !}+\ldots\right)$

Pour de très petites valeurs de $t_{1}$, on peut se limiter au premier terme de la série, et on obtient alors l'expression suivante :

$$
h_{\max }=b t_{1}
$$

Par contre, si l'on prend les deux premiers termes, on a :

$$
h_{\max }=b t_{1}\left(1-\frac{\alpha t_{1}}{2}\right)
$$

\section{c) INTÉgration DE L'ÉQuation COMPLÈte}

Pour la résolution de l'équation complète (15), on détermine les deux constantes $\mathrm{C}_{1}$ et $\mathrm{C}_{2}$ d'après les conditions suivantes :

$$
t=0 ; \quad y=\alpha ; \quad v=d y / d t=0
$$

Si l'on tient compte de (44) et (53), on obtient finalement, pour l'équation (15), la solution suivante :

$$
\begin{aligned}
& y=\frac{b e^{x_{1} t}}{\left(1-x_{1} / x_{2}\right)}+\frac{b e^{x_{2} t}}{x_{2}\left(1-x_{2} / x_{1}\right)}+\frac{\alpha}{\sqrt{1-4 \alpha \beta}} \\
& {\left[b\left(t / x_{1}+1 / x_{1}^{2}-t / x_{2}-1 / x_{2}{ }^{2}\right)+a \frac{\sqrt{1-4 \alpha \beta}}{\alpha}\right]}
\end{aligned}
$$

On a ici :

$$
\begin{aligned}
& x_{1}=\frac{-1+\sqrt{1-4 k^{2} / g l}}{2 k / g} \\
& x_{2}=\frac{-1-\sqrt{1-4 k^{2} / g l}}{2 k / g}
\end{aligned}
$$

De cette équation pour $y$ on déduit comme précédemment l'expression suivante pour la surpression hydraulique maximum au temps $t=t_{1}$.

$$
\begin{aligned}
h_{\mathrm{m}: \mathrm{tx}}=- & b\left(1 / x_{1}+1 / x_{2}\right) \\
& +\frac{b e^{x_{1} l_{1}}}{x_{1}\left(1-x_{1} / x_{2}\right)}+\frac{b e^{\cdot x_{2} l_{1}}}{x_{2}\left(1-x_{2} / x_{1}\right)}
\end{aligned}
$$

L'équation simplifiée (9) peut se déduire de l'équation (13) en admettant que $g=\infty$. Comme, dans ce cas, $x_{1}$ prend la valeur indéterminée $0 / 0$, son calcul nécessite un recours aux considérations de valeur limite suivantes. Si l'on écrit l'équation (13) sous la forme :

$$
\begin{gathered}
a_{2} y^{\prime \prime}+a_{1} y^{\prime}+a_{0} y=\rho /(x) \\
x_{1}=\left(-a_{1}+\sqrt{a_{1}^{2}-4 a_{0} a_{2}}\right) / 2 a_{2} \\
x_{22}=\left(-a_{1}-\sqrt{\left.a_{1}^{2}-4 a_{0} a_{2}\right)} / 2 a_{2}\right.
\end{gathered}
$$

Pour $g=\infty$, on a $a_{2}=0$.

On cherche la valeur limite :

$\lim _{a_{2}=0} \frac{-a_{1}+\sqrt{a_{1}^{2}}-4 a_{0} a_{2}}{2 a_{2}}=-\alpha=-k / l$

puisque :

$$
a_{0}=k / l \quad a_{1}=1 \quad a_{2}=k / g
$$

Il en résulte :

$$
x_{2}=-2 a_{1} / 0=-\infty
$$

En introduisant ces valeurs dans l'équation (15), on retrouve effectivement l'équation (12).

\section{d) Exemple 1}

Mais la constante $a_{2}$ s'annule aussi si la perméabilité $J$ est très petite. L'équation complète ne fournit donc un résultat numérique essentiellement différent de l'équation simplifiée, que si le sol présente une perméabilité suffisamment grande. Afin de juger s'il existe dans la pratique des perméabilités suffisamment grandes, on adoptera dans l'exemple suivant la perméabilité très élevée $k=2.10^{-4} \mathrm{~m} / \mathrm{s}\left(10^{-2} \mathrm{~m} / \mathrm{s}\right.$ représente pratiquement une limite supérieure correspondant à un sol sablonneux). Par ailleurs, supposons quc les conditions soient les suivantes :

Le plan d'eau maximum dans un port est à : $+4,28 \mathrm{~m}$. Il s'abaisse dans le temps $t_{1}=2$ jours $=1,73 \cdot 10^{-5} \mathrm{sec}$ à la cote la plus basse $\left(a-b t_{1}\right)$ $=0,50 \mathrm{~m}$ (fig. 24). Le trajet d'infiltration au-dessous de l'ouvrage (fig. 1, et 2) a pour. Iongueur $l$ $=33,5 \mathrm{~m}$. On peut calćuler la surpression hy-

(12) SchLercher. - Taschenbuch für Batingenieure, Berlin, 1943, p. 34 . 
dranlique d'apris ces données. On obtient pour' l'équation simplifiée el avec les valeurs suivantes:

$$
\begin{aligned}
b t_{1} & =4,28+0,50=4,78 \mathrm{~m} \\
\alpha & =k / l=0,6.10^{-5}(1 / \mathrm{sec}) \\
\mathrm{A} & =\alpha t_{1} / 2=0,5175
\end{aligned}
$$

différentes solutions suivant qu'on utilise les approximations indiquées ou la formule précise. II résulte alors de l'équation (46) :

$$
h_{\max }=4,78 / 1,5175=3,15 \mathrm{~m}
$$

Si, au lieu de l'équation approchée, on prend l'ćquation précise (49), on trouve :

$$
\begin{aligned}
b & =4,78 / 1,73 \cdot 10^{-5}-2,76 \cdot 10^{-5} \mathrm{~m} / \mathrm{s} \\
h_{\max } & =\frac{2,76 \cdot 10^{-5}}{0,60 \cdot 10^{-5}}\left(1-c^{-1,035}\right)=2,97 \mathrm{~m}
\end{aligned}
$$

Par conséquent, eu égard à l'imprécision inhérente à tout le calcul, la précision des résultats obtenus avec l'équation (46) est relativement bonne. Par contre, il n'est pas admissible d'utiliser l'équation (52) comme expression approchée dans le cas présent, car la valeur $t_{1}$ est trop grande pour cela. On obtiendrait :

$$
h_{\max }=4,78(1-0,5175)=2,31 \mathrm{~m}
$$

c'est-à-dire une valeur considérablement plus faible.

\section{e) Exrmure 2}

Afin de montrer l'influence sur la surpression de la vitesse d'abaissement du niveau libre, on prendra le même exemple que ci-dessus, mais avec la difrérence que le temps $t_{1}$ n'est plus deux jours, mais seulement 6 minutes. Ceci correspondrait par exemple, non plus à un port, mais à une écluse. On a : $t_{1}=6 \mathrm{~min}=360 \mathrm{sec}$. On a, de plus :

$$
\mathrm{A}=\alpha t_{1} / 2=1,08 \cdot 10^{-3}
$$

On trouve, pour la surpression, suivant l'équation (46) :

$$
h=4,78 / 1,0011=-4,78 \mathrm{~m}
$$

Ensuite, étant donné que

$$
b=4,78 / 360=0,0133 \mathrm{~m} / \mathrm{sec} .
$$

il résulte de l'équation (50):

$h_{\max }=\frac{0,0133}{0,6.10^{-i}}(0,00216-0,00000466+\ldots)=4,78 \mathrm{~m}$

Il suffit, dans le second cas, d'appliquer la formule approchée (51). Ceci signifie que le niveau de la nappe reste à la cote a du plan d'eau ini- tial. Autrement dit, la surpression a tout simplement pour valeur les $4,78 \mathrm{~m}$ correspondant à l'abaissement du niveau libre. C'est le cas le plus défavorable qui puisse en général se produire.

Si on utilise l'équation complète (56), il faut d'abord détermincr les facteurs $x_{1}$ et $x_{2}$. Dans les deux cas on trouve :

$\sqrt{1-4 k^{2} / g} l=\sqrt{1-4.4 .10^{-8} / 33,5.9,81} \equiv$ env. 1

Il faut donc employer pour $x_{1}$ la valeur limite qui est égale à - - éq. $57 a$ ).

Pour $\%$, on obtient, avec la valeur de $k$ indiquée ci-dessus, l'expression $\kappa_{2}=-g / k$. Il en

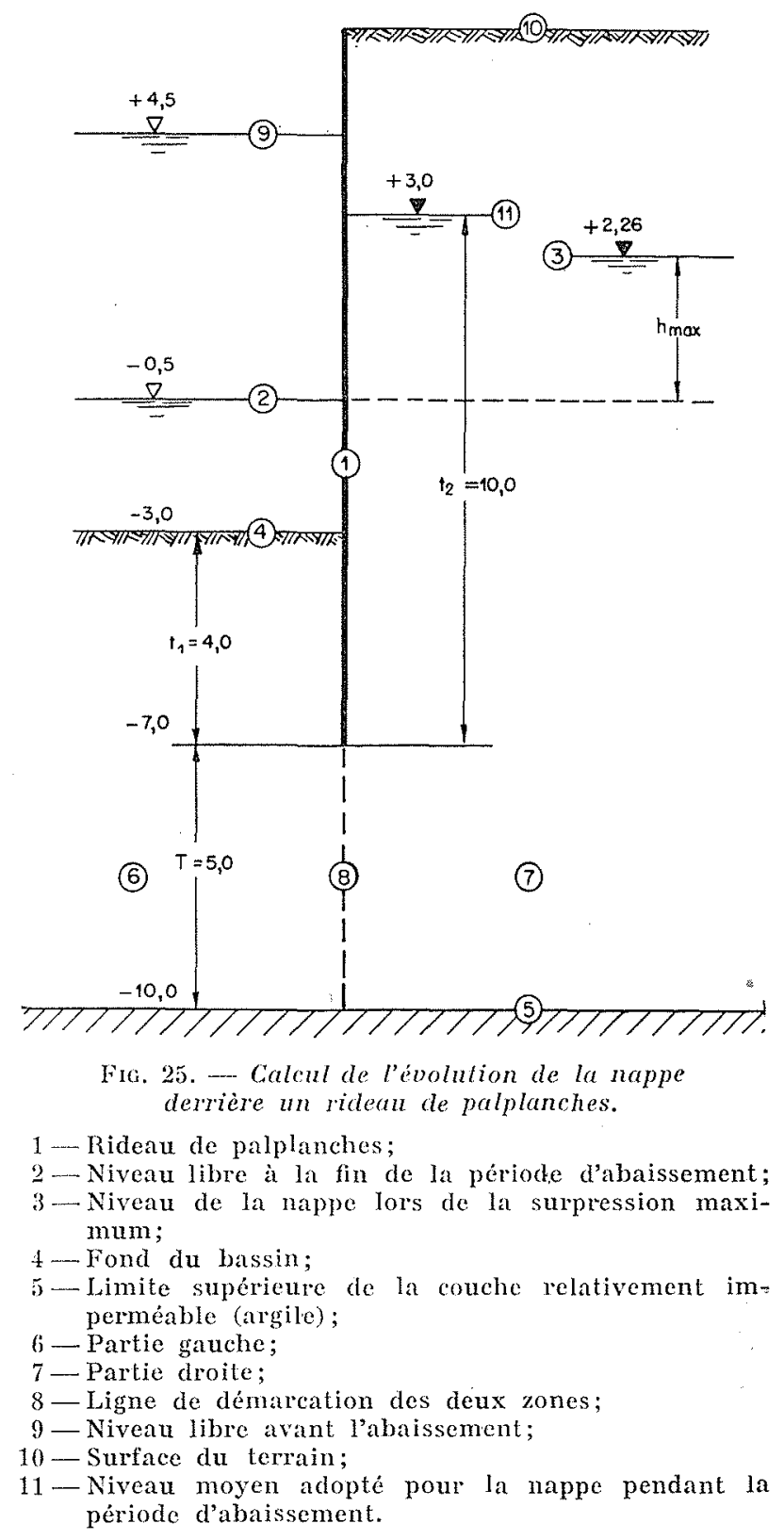


résulte pour la surpression hydraulique maximum :

$$
\begin{aligned}
h_{\max }=b\left(\frac{l}{k}+\frac{k}{g}\right)- & \frac{b e^{-(k / l) l_{1}}}{k / l\left(1-k^{2} / l g\right)} \\
& -\frac{b e^{-(g / k) t_{1}}}{g / k\left(1-k^{2} / g l\right)}
\end{aligned}
$$

Cette équation est valable pour les grandes valeurs de $k$, en admettant qu'on puisse poser avec une précision suffisante $\vee 1-4 k^{2} / g l=1$. C'est le cas pour toutes les valeurs de k que l'on rencontre. Dans le premier exemple, où $t_{1}$ était srand, on trouverait de plus :

$$
\%_{2} t_{1}=g t_{1} / k=8,49 \cdot 10^{9}
$$

Comme on a, en outre :

$$
k^{2} / g l=0,012210^{-8}=\text { environ } 0
$$

le dernier terme de l'équation (58) est également nul. L'expression $k / g$ devient aussi extraordinairement petite et égale à environ $0,2 \cdot 10^{-4}$, de sorte que l'équation complète perd tous les termes par lesquels elle diffère de l'équation simplifièe. L'équation simplifiée (12) suffit donc parfaitement, comme dans le cas du mouvement périodique, a la détermination de la surpression hydraulique pour les valeurs de $k$ que lon rencontre dans la pratique.

\section{1) ExEMrte: 3}

Alin de se rendre compte dans quelle mesure les résultats varient lorsque l'on considere le ré-

seau isotherme au lieu du tuyau, on va calculer l'exemple suivant.

Il s'agit d'un rideau de palplanches ayant les dimensions indiquées sur la figure 25. Supposons que, comme dans l'exemple précédent, on abaisse le niveau libre maximum de $5 \mathrm{~m}$ en deux jours. La perméabilité est encore $k=2 \cdot 10^{-1} \mathrm{~m} / \mathrm{s}$.

On a alors :

$$
b t_{1}=5,0
$$

Pour le tuyau, on a :

$$
l_{1}=t_{1}+t_{2}=4,0+10,0=14,0 \mathrm{~m}
$$

On admet ici que, pendant l'abaissement, le niveau moyen de la nappe [voir fig. 1 (5) et fig. 2 (5)] se trouve a la cote $+3,00$.

Etant donmé que $t_{2}$ est plus grand que ' $\mathrm{T}$, l'equation (28) ne s'applique plus exactement. Si on l'emploie quand mème, on a :

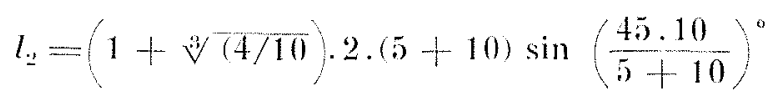

$$
\begin{aligned}
& =1,737.2 .15 \sin 30^{\circ}=26.0 \mathrm{~m}
\end{aligned}
$$

Mais on fait un calcul plus correct si l'on emploie l'equation (22) avec :

$$
F_{i}=\frac{\pi}{4-u_{m}}
$$

$$
\psi_{m}=\ln \frac{2 \sin \frac{\pi}{4}\left(1-\frac{t_{i}}{t_{i}+\mathrm{T}}\right)}{1+2 \cos \frac{\pi}{4}\left(1-\frac{t_{i}}{t_{i}+\mathrm{T}}\right)+\sqrt{\cos \frac{\pi}{2}}\left(1-\frac{t_{i}}{t_{i}+\mathrm{T}}\right)}
$$

(d'apres Dachlér, p. 65).

$i$ devant etre pris égal à 1 et $2, F_{n}=F_{\%}$. Pour $f_{0}(z)$, DAchler n'a pas établi d'expression, de sorte qu'un calcul ultérieur de forme simple n'est pas possible.

Par contre, si l'on a ' $\mathrm{T}=30 \mathrm{~m}, t_{2}$ est plus petit que T et l'équation (28) s'écrit :

$$
l_{: 2}=1,737.2(30+10) \sin \left(\frac{45.10}{30+10}\right)=27,1 \mathrm{~m}
$$

Par conséquent, la différence par rapport au tuyau est considérable. Elle est d'autant plus faible que T est plus petit de sorte que, plus la section au-dessous du rideau de palplanches est étroite, plus le calcul avec le tuyau est cxact.

On trouve de plus, pour le tuyau :

$$
x_{1}=k / l=1,43.10^{-5}(1 / \mathrm{s}) \text { et } x_{1} t_{1}=2,475
$$

pour les lignes de courant avee $\mathrm{T}=5 \mathrm{~m}$.

$$
\begin{gathered}
\mu_{2}=0,77.10 \pi(1 / \mathrm{s}) \text { et } \%_{2} t_{1}=1,32 \\
b=2,89.10 \pi \mathrm{m} / \mathrm{s}
\end{gathered}
$$

Il en résulte, d'après l'équation (49) :

$$
\begin{aligned}
& h_{1 \mathrm{max}}=\frac{2,89 \cdot 10^{-\pi}}{1,43 \cdot 10^{-\pi}}\left(1-e^{2,1 \pi i 5}\right) \\
& =\frac{2,89}{1,43}(1-0,085)=1,85 \mathrm{~m} \\
& h_{2 \mathrm{~m} \text { In: } \mathrm{x}}=\frac{2,89}{0,77}\left(1-e^{-1,3: 3)}\right. \\
& =\frac{2,89}{0,77}(1-0,264)=2,76 \mathrm{~m}
\end{aligned}
$$




\section{V. - RESUMÉ}

Le problème des surpressions derriere un mur de quai se ramène à l'étude des fluctuations de pression dans la nappe derrière le mur, à partir d'une loi donnée de variation du niveau libre. Nous avons étudié les fluctuations de la nappe en nous limitant au problème à deux dimensions et en supposant que, de part et d'autre de l'ouvrage, les écoulements sont surtout verticaux, autrement dit en supposant que les terrains avoisinants n'alimentent ni ne drainent la nappe. Nous avons déterminé la solution de l'équation différentielle fondamentale d'abord dans le cas de la marée (marée sinusoïdale), ensuite dans le cas d'un quai en rivicre (abaissement brusque du plan d'eau). Un certain nombre de conclusions générales peuvent être déduites de ces solutions, en particulier les conditions dans lesquelles on peut s'attendre a trouver des fluctuations de pression derrière le mur. La comparaison avec les observations faites dans la nature montre dans quelle mesure les hypotheses du calcul sont vérifiées et quels sont les écarts à prévoir par rapport à ces hypothèses. D'autres observations ont éte faites récemment et devront faire l'objet d'une étude séparée.

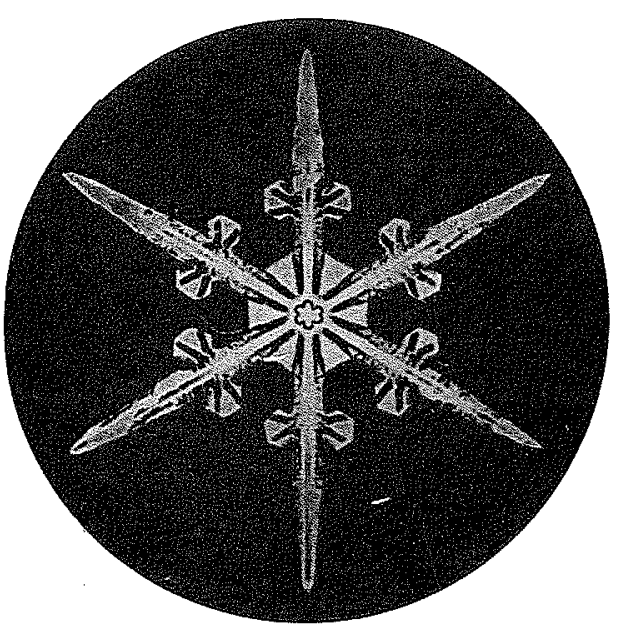

